\title{
Stress-impaired transcription factor expression and insulin secretion in transplanted human islets
}

\author{
Chunhua Dai, ${ }^{1}$ Nora S. Kayton, ${ }^{2}$ Alena Shostak, ${ }^{1}$ Greg Poffenberger, ${ }^{1}$ Holly A. Cyphert, ${ }^{2}$ Radhika Aramandla, ${ }^{1}$ \\ Courtney Thompson, ${ }^{1}$ Ioannis G. Papagiannis, ${ }^{1}$ Christopher Emfinger, ${ }^{1}$ Masakazu Shiota, ${ }^{2}$ John M. Stafford, ${ }^{1,2,3}$ \\ Dale L. Greiner, ${ }^{4}$ Pedro L. Herrera, ${ }^{5}$ Leonard D. Shultz, ${ }^{6}$ Roland Stein, ${ }^{2}$ and Alvin C. Powers ${ }^{1,2,3}$ \\ 'Division of Diabetes, Endocrinology, and Metabolism, Department of Medicine, and ²Department of Molecular Physiology and Biophysics, Vanderbilt University Medical Center, Nashville, Tennessee, USA. \\ ${ }^{3}$ VA Tennessee Valley Healthcare System, Nashville, Tennessee, USA. ${ }^{4}$ Department of Molecular Medicine, Diabetes Center of Excellence, University of Massachusetts Medical School, Worcester, \\ Massachusetts, USA. University of Geneva, Faculty of Medicine, Department of Cenetic Medicine and Development, Institute of Genetics and Genomics of Geneva and Centre Facultaire du Diabète, \\ Geneva, Switzerland. ${ }^{6}$ The Jackson Laboratory, Bar Harbor, Maine, USA.
}

\begin{abstract}
Type 2 diabetes is characterized by insulin resistance, hyperglycemia, and progressive $\beta$ cell dysfunction. Excess glucose and lipid impair $\beta$ cell function in islet cell lines, cultured rodent and human islets, and in vivo rodent models. Here, we examined the mechanistic consequences of glucotoxic and lipotoxic conditions on human islets in vivo and developed and/or used 3 complementary models that allowed comparison of the effects of hyperglycemic and/or insulin-resistant metabolic stress conditions on human and mouse islets, which responded quite differently to these challenges. Hyperglycemia and/or insulin resistance impaired insulin secretion only from human islets in vivo. In human grafts, chronic insulin resistance decreased antioxidant enzyme expression and increased superoxide and amyloid formation. In human islet grafts, expression of transcription factors NKX6.1 and MAFB was decreased by chronic insulin resistance, but only MAFB decreased under chronic hyperglycemia. Knockdown of NKX6.1 or MAFB expression in a human $\beta$ cell line recapitulated the insulin secretion defect seen in vivo. Contrary to rodent islet studies, neither insulin resistance nor hyperglycemia led to human $\beta$ cell proliferation or apoptosis. These results demonstrate profound differences in how excess glucose or lipid influence mouse and human insulin secretion and $\beta$ cell activity and show that reduced expression of key islet-enriched transcription factors is an important mediator of glucotoxicity and lipotoxicity.
\end{abstract}

\section{Introduction}

Patients with type 2 diabetes (T2D) have impaired insulin secretion in response to glucose, and this $\beta$ cell dysfunction is progressive, often requiring exogenous insulin therapy. Physiological levels of glucose and lipid stimulate insulin secretion. In excess, however, these nutrients are thought to directly impair insulin secretion and other aspects of $\beta$ cell function and survival, a phenomenon often referred to as glucotoxicity, lipotoxicity, and glucolipotoxicity, indicating the pathological consequences of excess glucose and/or lipid (1-3). Glucotoxicity and lipotoxicity are widely regarded as important contributors to the progressive decline of $\beta$ cell function in T2D.

Using rodent cell lines $(4,5)$, cultured rodent and human islets $(6,7)$, and in vivo rodent models $(8,9)$, investigators have suggested that excess glucose and/or lipid reduce insulin gene transcription (4), insulin protein content, glucose-stimulated insulin secretion (GSIS) $(10,11)$, and exocytotic events $(5,7)$. Use of somatostatin to "rest" $\beta$ cells by halting insulin secretion does not reverse or prevent these effects, suggesting that these toxicities are not simply due to insulin depletion (12). Increased islet amyloid deposition, which is associated with $\beta$ cell dysfunction and

Authorship note: C. Dai and N.S. Kayton contributed equally to this work. Conflict of interest: The authors have declared that no conflict of interest exists. Submitted: July 10, 2015; Accepted: February 24, 2016.

Reference information: J Clin Invest. 2016;126(5):1857-1870. doi:10.1172/JCI83657. apoptosis in T2D patients $(13,14)$, is also a proposed consequence of excess glucose and/or lipid $(15,16)$. Such circumstances promote rodent $\beta$ cell apoptosis $(17,18)$. Based on in vitro studies, the lipid contribution to apoptosis depends on the lipid species, with saturated fatty acids promoting apoptosis (19), potentially through ceramide formation $(20,21)$, altered lipid partitioning $(22-24)$, or oxidative stress $(2,25-27)$.

Notably, high glucose and/or lipid levels reduce the expression and function of transcription factors critical to $\beta$ cell development and function in cultured islets or in vivo rodent T2D models, particularly MAFA, NKX6.1, and PDX1 $(13,14,28)$. In fact, transgenic misexpression of MAFA is able to partially rescue many islet $\beta$ cell deficiencies in $d b / d b$ mice, a model of T2D (29). Moreover, MAFA, MAFB, NKX6.1, and PDX1 were also selectively lost in human T2D islets $(17,18,28)$; MAFA and MAFB are only coproduced in human islet $\beta$ cells (30). Due to the relative sensitivity of these transcription factors to T2D stressors and their established role in regulating mouse islet cell function, it was proposed that MAFA and/or MAFB is compromised early and that overt changes in $\beta$ cell dysfunction/ death reflect subsequent loss of NKX6.1 and/or PDX1.

Mechanistic studies of human islets in vivo are difficult to perform. However, alternative approaches using islet cell lines and islets in culture do not mimic islet regulation in vivo. Although cultured islets contain some endothelial and nerve cells, they lack integrated, functional vascularization and innervation, which is 
reestablished upon transplantation (31). Moreover, culturing islets leads to changes in islet gene expression (32). Furthermore, such in vitro studies are challenged by selection of individual lipid species, lipid concentrations, and/or glucose concentrations. Rodent models of T2D, such as the ZDF rat or $d b / d b$ mouse, also do not allow one to differentiate the effects of hyperglycemia from those of hyperlipidemia. In addition, it is possible that human islets have a unique response to these stress conditions in relation to mouse islets because there are fundamental differences in (for example) islet architecture $(19,33,34)$, islet-cell transcription factor composition $(20,21,35)$, and proliferative capacity (22-24).

As a result of these experimental limitations and species differences, the mechanisms of how excess glucose and/or lipid specifically impair human islet function in vivo are incompletely understood. To directly address if and how excess glucose and/or lipid levels differentially impair human verus mouse islet function, we generated or used models of metabolic stress in which engrafted human and mouse islets are exposed to hyperglycemia (i.e., glucotoxicity) and/ or insulin resistance (i.e., characterized by excess lipid [lipotoxicity]) and examined the in vivo impact on insulin secretion, oxidative stress, transcription factor expression, the unfolded protein response (UPR), proliferation, apoptosis, and amyloid deposition.

\section{Results}

To examine the consequences of hyperglycemia and/or excess lipid on mammalian islets in vivo, we developed and characterized animal models involving transplanted human and/or mouse islets exposed to chronic hyperglycemia (NSG-DTR model), chronic insulin resistance (NSG-HFD), or acute hyperglycemia and acute insulin resistance (NSG-S961) (Figure 1, A and B). Each model capitalizes on the profound immunodeficiency of the NOD.Cg-

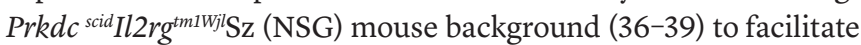
islet engraftment and employs preexperimental assessment of islet function to ensure islet quality (Figure 1C). Importantly, these models also allowed comparison of the in vivo response of human and mouse islets to these metabolic stresses.

Chronic hyperglycemia model (NSG-DTR). To directly examine the effect of chronic hyperglycemia on human islets in vivo, we developed a model in which one can specifically ablate native mouse pancreatic $\beta$ cells without harming transplanted human islets, which engrafted under normoglycemic conditions. To ablate mouse $\beta$ cells, we used the RIP-DTR mouse, in which human diphtheria toxin receptor (DTR) expression is controlled by the rat insulin promoter (RIP) (40). The RIP-DTR mouse was crossed onto the NSG background to produce the NSG-DTR mouse, a severely immunodeficient mouse with excellent xenograft tolerance, in which diphtheria toxin (DT) injection can ablate mouse $\beta$ cells (Supplemental Figure 1, A, E, and F;; supplemental material available online with this article; doi:10.1172/JCI83657DS1). We examined the response of NSG-DTR mice to a range of DT doses. A single injection of $5 \mathrm{ng}$ DT rapidly generated extreme and persisting hyperglycemia (Supplemental Figure 1B) and dramatically reduced both mouse pancreatic insulin content (Supplemental Figure 1C) and islet size (Supplemental Figure 1, E and F). Human islets were examined at the $5 \mathrm{ng}$ DT dose, and there was no overt effect on transplanted human islet function, insulin content, or islet survival (Supplemental Figure 1, D, G, and H).
We determined that 4,000 islet equivalent (IEQ) maintained normoglycemia in the majority of NSG-DTR+I mice (NSG-DTR mice with transplanted human islets) after DT-induced mouse $\beta$ cell ablation, but that most mice with only 2,000 IEQ quickly became hyperglycemic and remained so (Figure 1D). Notably, mouse pancreatic insulin content was greatly reduced in both of the DT-treated groups, DT-HG and DT-NG (Figure 1E). Mice were grouped for subsequent analysis based on their observed glycemic status rather than by the number of islets transplanted. Thus, we use the terms DT-HG (hyperglycemia after DT), DT-NG (normoglycemia after DT), and PBS (animals given PBS instead of DT) to describe the human islet transplanted groups.

Chronic insulin resistance model (NSG-HFD). A high-fat diet (HFD) was used to introduce excess dietary lipid and induce insulin resistance on the NSG background. Some mice exhibited high sensitivity to the diet (HFD-HS), and others exhibited low sensitivity (HFD-LS), as defined by the change in body weight and fat mass, glucose tolerance, and serum insulin (Supplemental Figures 2 and 3). Body weight (Supplemental Figure 2, A and B), percentage of fat and lean mass (Supplemental Figure 2, C-F), glucose tolerance (Supplemental Figure 3, A-D), and fasting serum insulin (Supplemental Figure 3, E-H) were affected on both $45 \%$ and $60 \%$ HFDs. However, only mice on a $60 \%$ HFD were subsequently used to test the effects of insulin resistance on human islets in vivo. We also generated and characterized 2 widely used genetic models of insulin resistance on the NSG background: the Glut4 ${ }^{-/}$(NSGGlut4) and the $o b$ (NSG-ob/ob). The phenotypes of Glut $^{-/-}$and $o b / o b$ mice on the NSG background (Supplemental Figures 5-7) differed considerably from those on the C57BL/6 background, so these models were not subsequently studied. These unexpected observations in the NSG-Glut4 and the NSG-ob/ob models exemplify how genetic background can affect the metabolic phenotype.

NSG mice with transplanted human islets were placed on the $60 \%$ HFD or regular diet (RD) (HFD+I and RD+I mice) for 12 weeks (Figure 1A), and this allowed a comparison of transplanted human islets and endogenous pancreatic mouse islets under the same metabolic conditions. One week before sacrifice (11 weeks on HFD), HFD+I mice had almost 3-fold greater weight gain (Figure $1 \mathrm{~F}$ and Supplemental Figure $4 \mathrm{~A}$ ), twice the percentage of fat mass (Figure $1 G$ ), and reduced lean mass (Supplemental Figure 4B) compared with RD+I controls. In addition, HFD+I mice had higher serum triglyceride and cholesterol levels (Figure 1H), mild hyperglycemia (Figure 1I), and glucose intolerance (Figure 1J). HFD+I mice had dramatic hepatic lipid deposition (Supplemental Figure 4C), and mouse islet size and $\beta$ cell mass were increased (Supplemental Figure 4, D and E), recapitulating prior studies on the effect of HFD on mouse islets (41-43).

We examined graft vessel morphology in human and mouse islet grafts in the NSG-HFD model to determine whether islet vasculature was affected by the HFD. Given that islet grafts revascularize with both donor and recipient endothelial cells, sometimes forming chimeric vessels (31), we stained with PECAM to detect mouse endothelial cells and with CD31, which identifies human endothelial cells. We found similar vessel morphology (size and density) in both diet groups, with human and mouse endothelial cells contributing to vessel formation (Supplemental Figure 8, A and B). By electron microscopy, we observed normal fenestration of human vessels on 
A

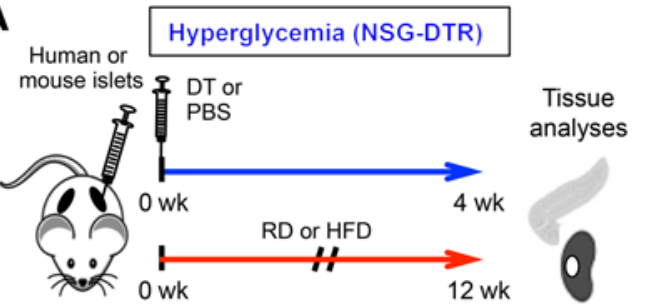

B

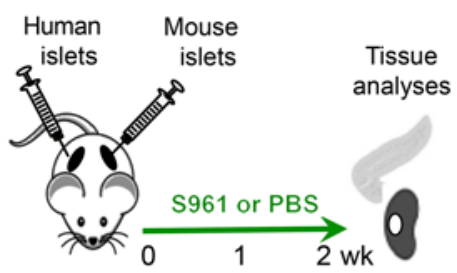

Dyslipidemia / Insulin Resistance (NSG-HFD)

C

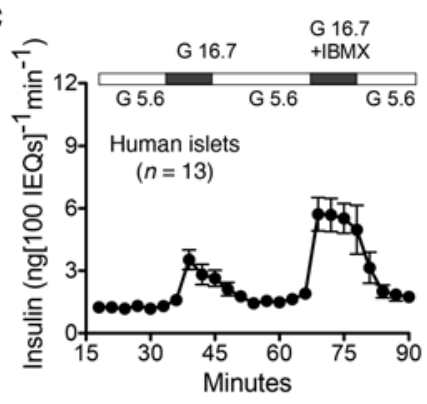

$\mathbf{F}$
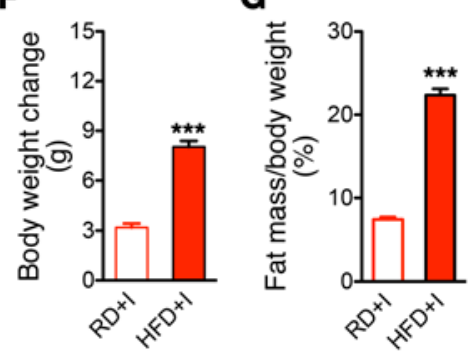

J

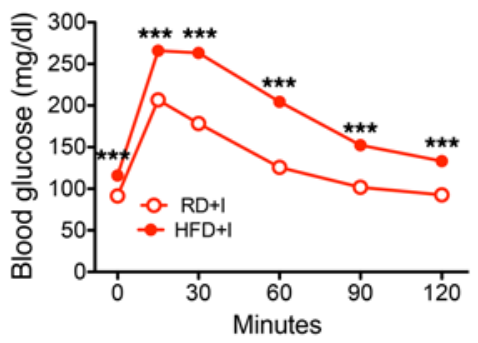

D

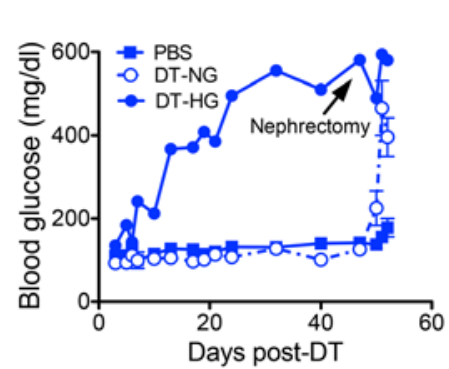

H

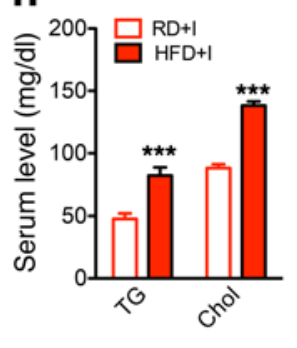

K

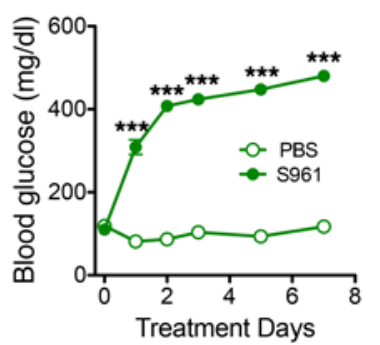

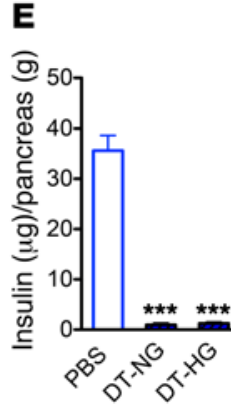

I
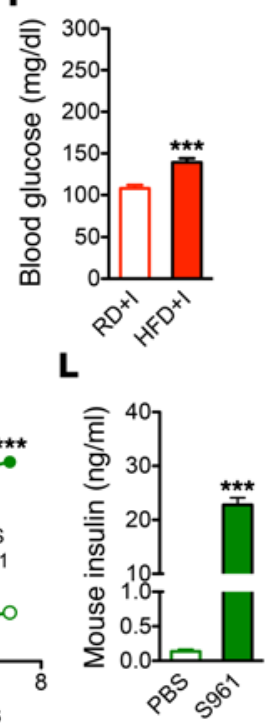

Figure 1. Models of chronic metabolic stress. (A) Experimental design. After islet engraftment period, NSG-DTR mice were injected with $5 \mathrm{ng}$ DT or saline and monitored for 4 weeks; NSC mice were placed on HFD or RD for 12 weeks. (B) Experimental timeline of $\mathbf{S 9 6 1}$ model. Two weeks after islet transplantation, S961 is delivered by implantation of osmotic pump. Analyses were performed at 1 or 2 weeks after pump implantation. (C) Isolated human islet preparations $(n=13)$ perifused, prior to transplantation, with media containing $5.6 \mathrm{mM}$ or $16.7 \mathrm{mM}$ glucose ( 5.6 and $\mathrm{G} 16.7$ ), then $16.7 \mathrm{mM}$ glucose with the phosphodiesterase inhibitor IBMX; some of these were part of previously published perifusion data sets $(35,102)$. (D) Random blood glucose of NSGDTR groups after DT injection ( $n=6$ / group). Nephrectomy indicates survival surgery to remove graft-containing kidney. (E) Pancreatic insulin content in NSG-DTR mice 4 weeks after DT injection ( $n=4-6 /$ group). ${ }^{* *} P<0.001$, DTNG or DT-HG vs. PBS. (F) Mouse body weight change after 12 weeks of diet (RD, $n=29$; HFD, $n=30$ ). ${ }^{* *} P<0.001$. (G) Fat mass (NSG-RD, $n=29$; NSG-HFD, $n=30$ ). (H) Serum triglyceride and cholesterol levels after 11 weeks on diet (NSG-RD, $n=8$; NSG-HFD, $n=9$ ). (I) Random blood glucose after 8 weeks of diet (RD, $n=20$; HFD, $n=21$ ) (J) Glucose tolerance test after 8 weeks on diet (NSG-RD, $n=31$; NSG-HFD, $n=33$ ). ${ }^{* *} P<0.001$. (K) Random blood glucose measurements of S961- and PBS-treated mice from 0 to 7 days after pump implantation. ${ }^{* *} P<0.001$. (L) Random (nonfasting) mouse insulin values. ${ }^{* *} P<0.001$. PBS, $n=8$; S961, $n=12$. Unpaired 2-tailed Student's $t$ test or 1-way ANOVA followed by Newman-Keuls multiple comparison test (E) was used for analysis of statistical significance. Blue represents the NSG-DTR model, red the NSG-HFD model, and green the NSG-S961 model. DT-HG, hyperglycemia after DT; DT-NG, normoglycemia after DT; PBS, animals given PBS instead of DT. both diets (Supplemental Figure 8, E and F). We found similar results in mouse islet grafts, with similar density, distribution, and size of vessels in both diet groups (Supplemental Figure 8, C, D, G, and H). Taken together, these data indicate that 12 weeks on HFD do not change the vasculature of transplanted human or mouse islet grafts.

Acute hyperglycemia and insulin-resistance model (NSG-S961). To examine the effect of a shorter duration of metabolic stress on human islets, we treated mice with the insulin receptor antagonist S961 (Figure 1B), a 43-aa peptide antagonist known to induce many consequences of insulin resistance in rodents, including hyperglycemia, hyperinsulinemia, decreased hepatic glycogen storage, and decreased adipocyte triglyceride storage $(44,45)$. In our studies, S961-treated mice became hyperglycemic 24 hours after injection (Figure 1K) and remained so at 2 weeks (Supplemental Figure 9A). The insulin resistance of these mice is illustrated by extreme hyperinsulinemia of both human (Supplemental Figure 9B) and mouse insulin (Figure 1L).
Metabolic stresses impair stimulated human insulin secretion in vivo. DT-HG, HFD+I, and S961-treated mice all showed hyperglycemia (Figure 2, A, E, and J, respectively) and fasting human hyperinsulinemia (Figure 2, B, F, and $\mathrm{K}$, respectively). Stimulated human insulin secretion dramatically decreased or was suppressed (DT-HG) in these groups (Figure 2, B, F, and K). The ratio of stimulated human insulin to blood glucose, a measure of $\beta$ cell responsiveness to hyperglycemia, was reduced in both DT-HG mice and HFD+I mice (Figure 2, C and G). In contrast, stimulated mouse insulin levels were quite elevated in HFD+I (Figure 2H) and unchanged in s961-treated mice (Figure 2L). This demonstrates a fundamental functional difference between mouse and human islets under identical metabolic stress conditions.

Insulin content of the human islet graft was unchanged in DT-HG and HFD+I mice (Figure 2, D and I), but it was markedly reduced in S961-treated mice (Figure $2 \mathrm{M}$ ). In contrast, content 

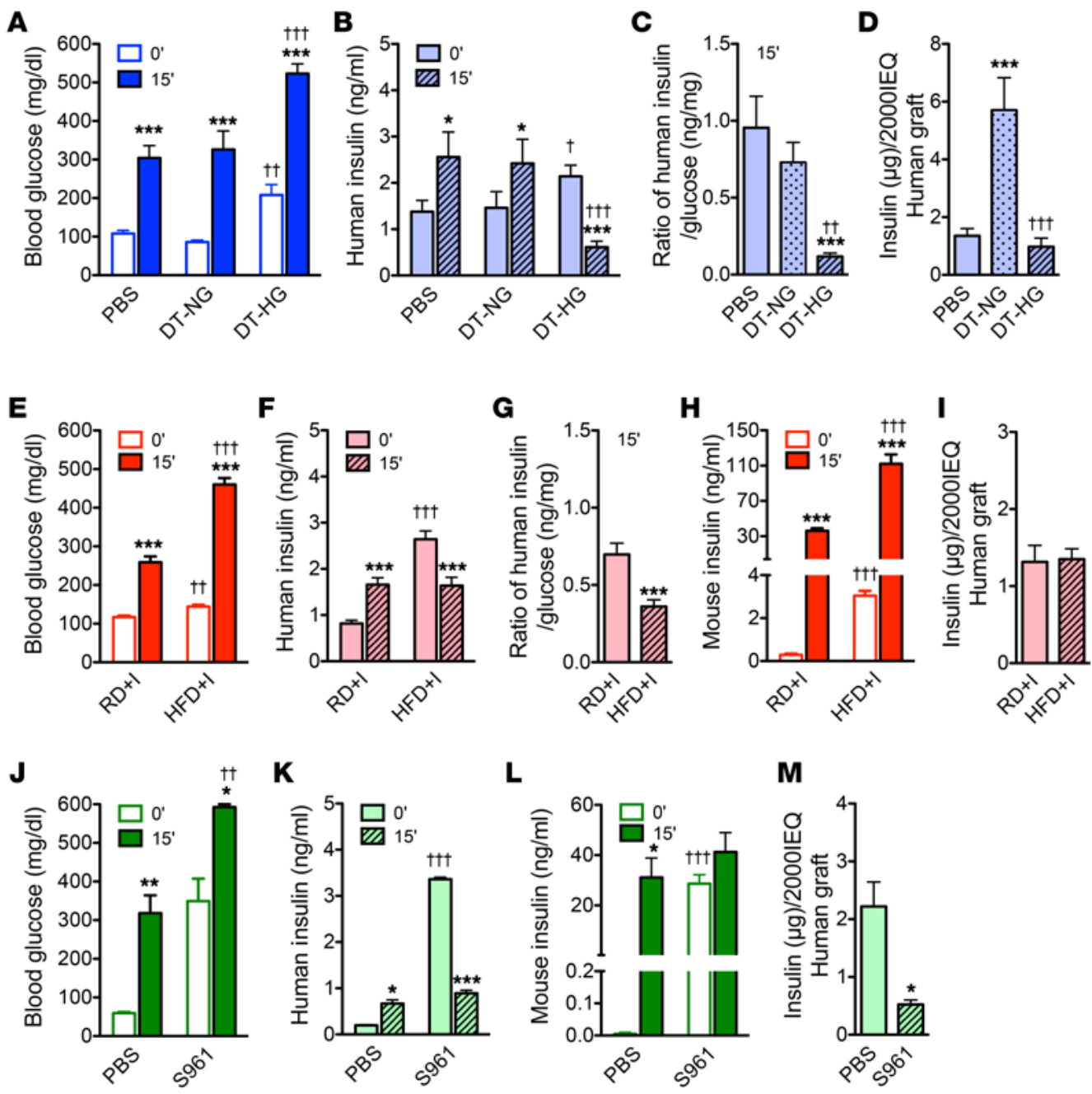

Figure 2. Chronic hyperglycemia, chronic insulin resistance, and acute hyperglycemia and insulin resistance impair stimulated insulin secretion from transplanted human $\beta$ cells. (A) Blood glucose of DTR groups after 6 -hour fast $\left(\mathrm{O}^{\prime}\right)$ and 15 minutes after injection of glucose $(2 \mathrm{~g} / \mathrm{kg})$ plus arginine $(2 \mathrm{~g} / \mathrm{kg}){ }^{* * *} P<0.001$, $\mathrm{O}^{\prime}$ vs. $15^{\prime}$ within each group; ${ }^{\dagger+} P<0.01$, DT-HG O' vs. PBS $0^{\prime} ;{ }^{+t+} P<0.001$, DT-HG $15^{\prime}$ vs. PBS $15^{\prime}$. (PBS, $n=15$; DT-NG, $n=13 ;$ DT-HG, $n=17$ ). (B) Human insulin secretion from glucose-arginine stimulation assay. ${ }^{*} P<0.05,{ }^{* * *} P<0.001, \mathrm{O}^{\prime}$ vs. $15^{\prime}$ within each group; ${ }^{\dagger} P<0.05, \mathrm{DT}-\mathrm{HG} \mathrm{O}^{\prime}$ vs. PBS $\mathrm{O}^{\prime} ;{ }^{\prime+t} P<0.001, \mathrm{DT}-\mathrm{HG} 15^{\prime}$ vs. PBS 15' (PBS, $n=15$; DT-NG, $n=13$; DT-HG, $n=17$ ). (C) Data expressed as ratio of human insulin (data from B) to blood glucose level (data from A). ${ }^{* * *} P<0.001$, DT-HG vs. PBS; ${ }^{\dagger+} P<0.01$, DT-HG vs. DT-NG. (D) Human graft insulin content. ${ }^{* * *} P<0.001$, DT-NG vs. PBS, ${ }^{+t+} P<0.001, \mathrm{DT}-\mathrm{HG}$ vs. DT-NG $(n=5-6 /$ group). (E-H) Glucose-arginine stimulation of HFD model after 11 weeks on diet. (E) Blood glucose values. ${ }^{* * *} P<0.001,0^{\prime}$ vs. $15^{\prime}$ within the each diet group; ${ }^{t+} P<0.01, O^{\prime}$ vs. $O^{\prime}$ between 2 diet groups; ${ }^{t+t} P<0.001,15^{\prime}$ vs. $15^{\prime}$ between 2 diet groups (NSG-RD, $n=27 ; \mathrm{HFD} ; n=34$ ). (F) Human and (H) mouse serum insulin levels. ${ }^{* * *} P<0.001, O^{\prime}$ vs. $15^{\prime}$ within the each diet group; ${ }^{+{ }^{+t}} P<0.001, O^{\prime}$ vs. $O^{\prime}$ or $15^{\prime}$ vs. $15^{\prime}$ between 2 diet groups (NSG-RD, $n=27 ;$ NSG-HFD; $n=34$ ). (C) Data expressed as ratio of human insulin (data from F) to blood glucose level (data from E). ${ }^{* *} P<0.001$. (I) Human graft insulin content. $P=0.880$ (NSG-RD, $n=12$; NSG-HFD; $n=14)$. (J-L) Glucose-arginine stimulation of S961 model, 10 days after injection. (J) Blood glucose values, (K) human insulin secretion, (L) mouse insulin secretion. ${ }^{*} P<0.05,{ }^{* *} P<0.01,{ }^{* * *} P<0.001,0^{\prime}$ vs. $15^{\prime}$ within the each treatment; ${ }^{\dagger+} P<0.01,{ }^{t+t} P<0.001,0^{\prime}$ vs. $0^{\prime}$ or $15^{\prime}$ vs. $15^{\prime}$ between 2 treatments ( $n=5$ /treatment). (M) Human graft insulin content. ${ }^{*} P<0.05$ ( $n=5$ /treatment). Unpaired 2-tailed Student's $t$ test or 1-way ANOVA followed by Newman-Keuls multiple comparison test ( $\mathbf{C}$ and $\mathbf{D}$ ) was used for analysis of statistical significance. Blue represents the NSG-DTR model, red the NSG-HFD model, and green the NSG-S961 model. Darker bars represent mouse data; lighter bars represent human data.

was increased 3-fold in DT-NG grafts (Figure 2D). Together, these results indicate that the conditions of chronic hyperglycemia, chronic insulin resistance, and acute hyperglycemia with insulin resistance impair human islet function in vivo, but do not have a similar affect on mouse insulin secretion.

Human $\beta$ cells do not proliferate in response to hyperglycemia or insulin resistance. Insulin resistance promotes compensatory expansion of rodent $\beta$ cell mass due to proliferation $(42,43,46)$, and glucose has been reported to be a rodent and human $\beta$ cell mitogen (47-49). As expected, native mouse pancreatic islets in
HFD+I mice (Supplemental Figure 4, F and G) and NSG-S961 mice (Supplemental Figure 9, C and D) showed an increased percentage of Ki67 ${ }^{+} \beta$ cells, reflecting induced proliferation. HFD+I mice also had larger islets and increased pancreatic $\beta$ cell mass (Supplemental Figure 4, D and E). Human $\beta$ cell proliferation was very low, and in stark contrast to mouse $\beta$ cells, it was unchanged by the condition of metabolic stress in each model (Figure 3, A-D, $\mathrm{H}$, and I). We use the term "proliferation" to refer to cells labeled with Ki67, while acknowledging that this is not a direct measure of proliferation as defined by an increase in $\beta$ cell number. 

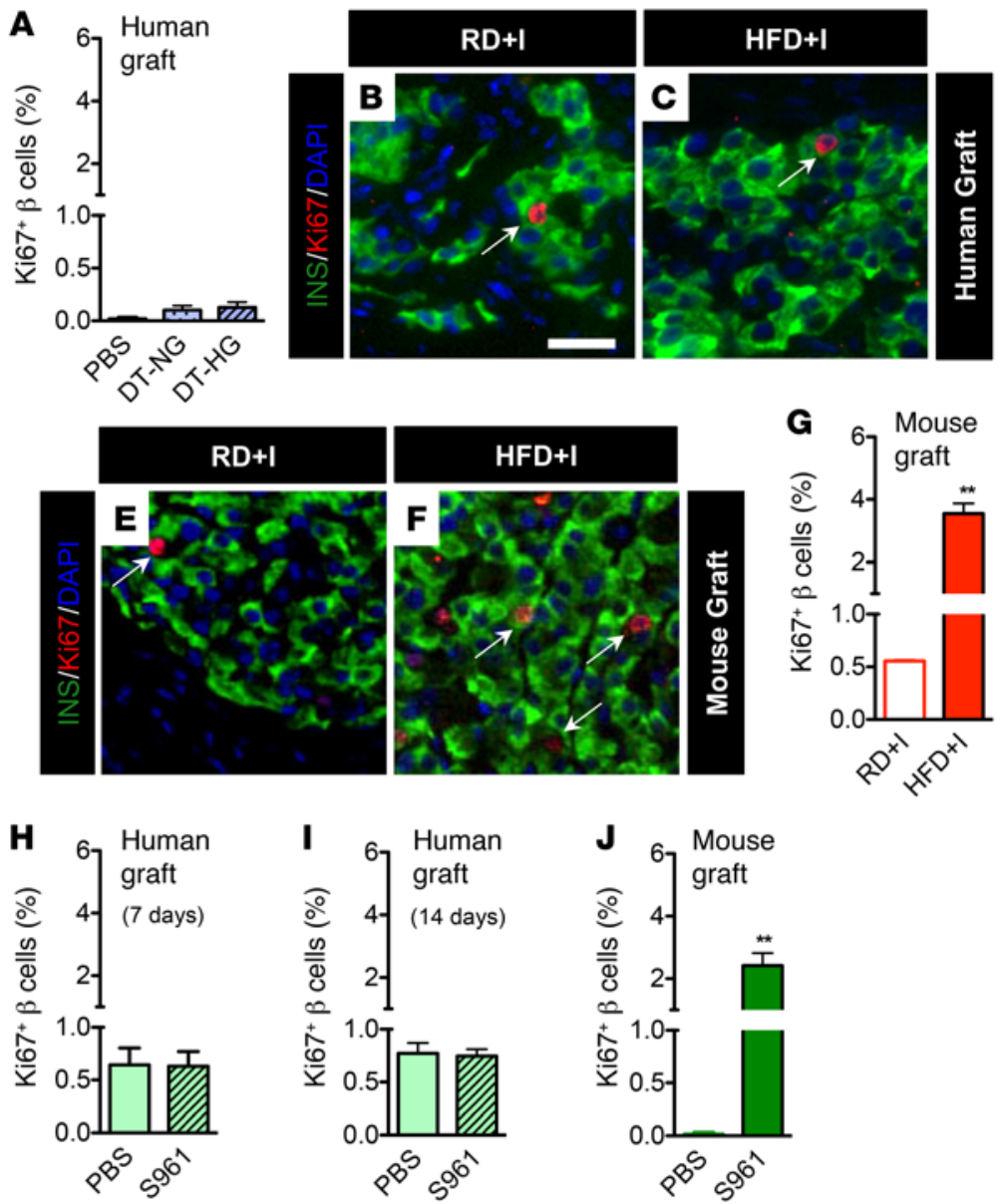

$\mathbf{K}$

Human graft

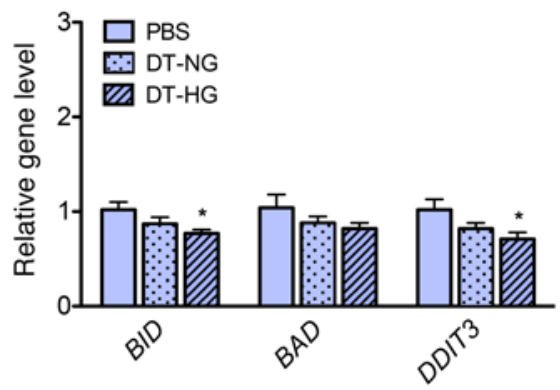

$\mathbf{L}$

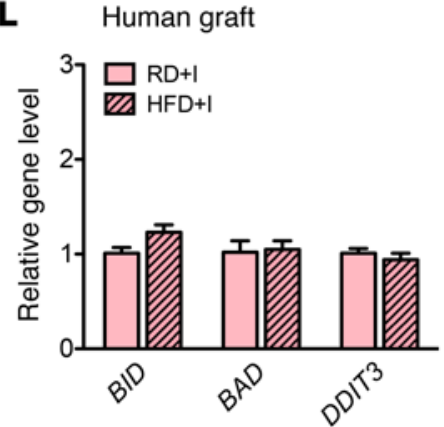

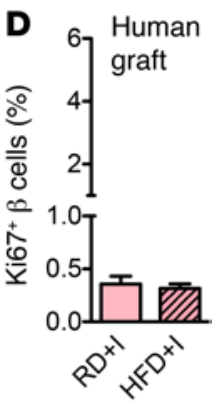

Figure 3. Human $\beta$ cells do not proliferate in response to hyperglycemia or insulin resistance. Quantification of $\beta$ cell proliferation in (A) NSG-DTR human grafts (PBS, $n=9$; DT-NG, $n=5$; DT-HG, $n=12$ ). (B-C) Images of NSG-HFD (and RD) human grafts. Scale bar: $25 \mu \mathrm{m}$. (D) Quantification of percentage of Ki67 $\beta$ cells in NSG-HFD human grafts. $n=11 /$ diet, $P=0.633$. (E and F) Images of NSG-HFD (and RD) mouse grafts. (G) Quantification of percentage of Ki67 $\beta$ cells in NSG-HFD mouse grafts ( $n=3 /$ diet). ${ }^{* *} P<0.01$. Islet images (B, $\mathbf{C}, \mathbf{E}$, and $\mathbf{F})$ labeled with insulin (green), Ki67 (red), and DAPI (blue). Arrows point to proliferating Ki67-positive $\beta$ cells. The number of $\beta$ cells counted in each group was 7,000 to 16,000 . $\beta$ cell proliferation in S961-treated $(\mathbf{H})$ human grafts (left kidney, $n=5 /$ treatment, $P=0.644$ ) after 7 days, (I) human grafts after 14 days ( $n=5 /$ treatment, $P=0.8239$ ), and $(J)$ contralateral mouse graft (right kidney, $n=5$ /treatment, ${ }^{* *} P<0.01$ ) after 7 days. ( $\mathbf{K}$ and $\mathbf{L}$ ) Expression of apoptosis-related genes $B I D, B A D$, and DDIT3 (CHOP) in human grafts (K) from NSG-DTR model (PBS, $n=5$, DT-NG, $n=5$; DT-HG, $n=10 ;{ }^{*} P<0.05$, DT-HG vs. PBS) and (L) from NSG-HFD model ( $n=5$ / diet; $P>0.05)$. Unpaired 2-tailed Student's $t$ test or 1-way ANOVA followed by NewmanKeuls multiple comparison test (K) was used for analysis of statistical significance. Blue represents the NSG-DTR model, red the NSG-HFD model, and green the NSG-S961 model. Darker bars represent mouse data; lighter bars represent human data.
To address the possibility that the difference between human and mouse $\beta$ cell proliferation in response to metabolic stress was related to the kidney capsule transplantation site, we transplanted NSG mice with mouse islets under the kidney capsule, prior to HFD or S961 treatment. Mouse graft $\beta$ cell proliferation increased more than 6-fold in HFD-fed mice (Figure 3, E-G) and increased nearly 20-fold in NSG-S961 mice (Figure 3J), results that are similar to those seen in other studies using S961 (50, 51). Importantly, these results demonstrate that the profound difference in proliferation observed between human and mouse islets is species specific and not an effect of the transplantation site.

Neither chronic hyperglycemia nor insulin resistance causes human $\beta$ cell apoptosis. Multiple in vitro studies have suggested that chronic metabolic stresses promote $\beta$ cell apoptosis and decrease islet survival $(17,18)$. To address whether human $\beta$ cell loss con- tributed to impaired stimulated insulin secretion, we measured expression of the key apoptosis genes $\mathrm{BH} 3$ interacting domain death agonist (BID), BCL2 associated agonist of cell death (BAD), and DNA damage inducible transcript 3 (DDIT3) (CHOP) in DTHG and HFD+I human grafts. Two markers of apoptosis were decreased in DT-HG grafts (Figure 3K), and all 3 were unchanged in HFD+I grafts (Figure 3L). The lack of increased CHOP expression in both models indicates that human $\beta$ cells under chronic hyperglycemia or chronic insulin resistance were not undergoing stress-induced apoptosis. Indeed, we observed only rare apoptotic $\beta$ cells in both mouse and human grafts in the HFD model (Supplemental Figure 10, A-F) and in human grafts in the DTR model (Supplemental Figure 10G) at rates similar to those seen with control grafts. Thus, excess glucose or lipid does not lead to apoptosis in human or mouse islets in vivo. 

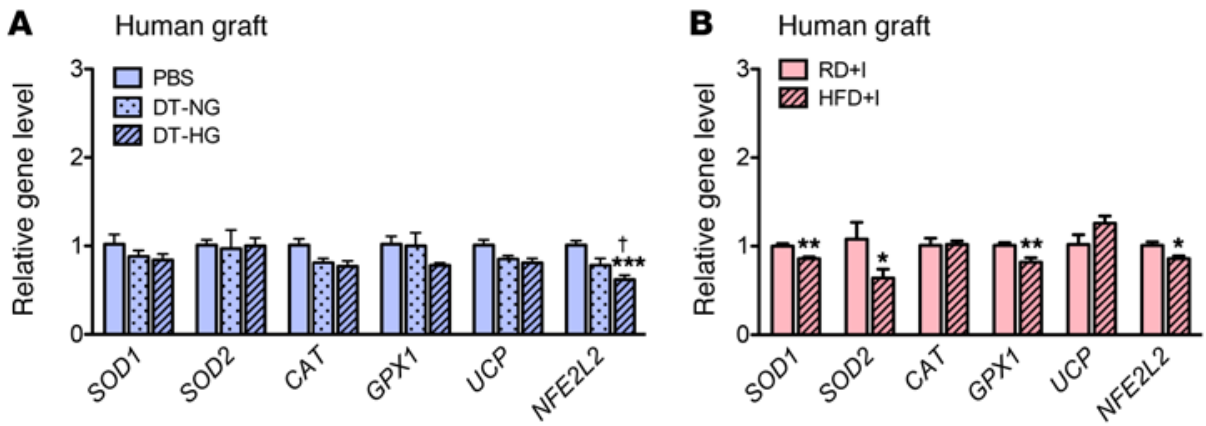

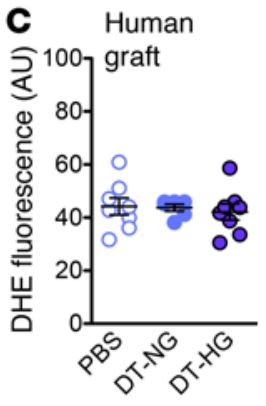

G

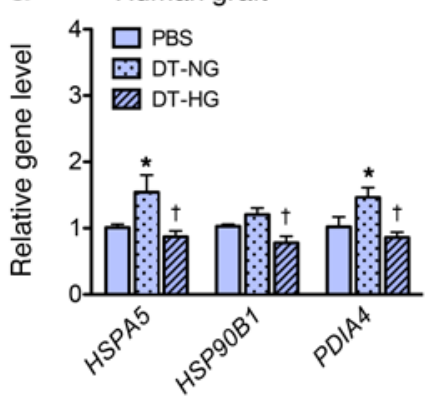

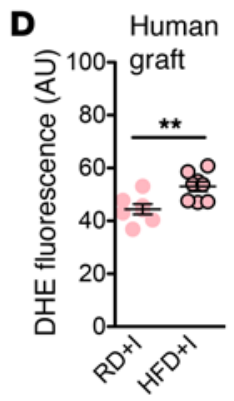

E
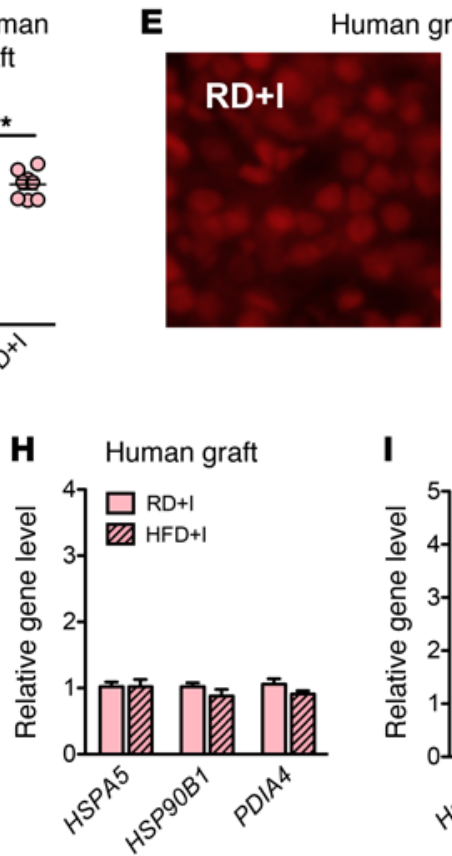

I Mouse pancreatic islet

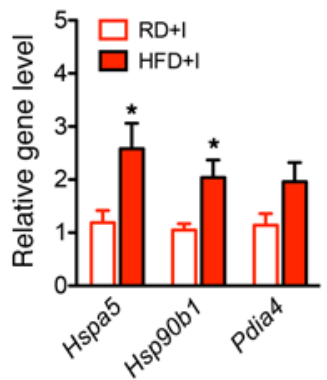

Figure 4. Antioxidant enzymes, ROS, and UPR. (A and B) Relative expression of antioxidant enzymes (SOD1, SOD2, CAT, GPX1, and UCP2) and oxidative stress-responding transcription factor (NFE2L2) gene in transplanted human islets from (A) NSG-DTR (PBS $n=5$, DT-NG, $n=5 ;$ DT-HG, $n=10 ;{ }^{* *}$ DT-HG vs. PBS, †DT-HG vs. DTR-NG) and (B) NSG- HFD $\left(n=5 /\right.$ diet; ${ }^{*} P<0.05$, $\left.{ }^{* *} P<0.01\right)$ models. Quantification of superoxide production, measured by fluorescence of DHE staining, in (C) NSG-DTR human grafts ( $n=6$-8/group), (D) NSG-HFD human grafts $\left(n=8 /\right.$ diet, $\left.{ }^{* *} P<0.01\right)$, and (F) HFD mouse grafts ( $n=7-8$ / diet). (E) Images of DHE staining in NSG-HFD/RD human grafts. UPR genes are induced in mouse islets and in DT-NG human grafts, but not in DT-HG or HFD+I human grafts. Original magnification, $\times 20$. (G-I) mRNA levels of UPR marker genes in (C) NSG-DTR human grafts (PBS, $n=5 ;$ DTR-NG, $n=5$; DTR-HG, $n=10$ ). ${ }^{*} P<0.05$, DT-NG vs. PBS; ${ }^{\dagger} P<0.05$, DT-HG vs. DT-NG. (H) NSG-HFD human grafts $\left(n=5 /\right.$ diet), and $(\mathrm{I})$ mouse islets $\left(n=5 /\right.$ diet). ${ }^{*} P<0.05$. Unpaired 2-tailed Student's $t$ test or 1-way ANOVA followed by Newman-Keuls multiple comparison test (A and $\mathbf{G}$ ) was used for analysis of statistical significance. Blue represents the NSG-DTR model; red represents the NSG-HFD model. Darker bars represent mouse data; lighter bars represent human data.

Chronic hyperglycemia or chronic insulin resistance decreases antioxidant enzyme expression and increases superoxide levels in human islet grafts. Oxidative stress from increased levels of ROS is widely hypothesized as a cause of $\beta$ cell dysfunction $(2,52)$. These cells are thought to be more sensitive to ROS due to their unusually low levels of antioxidant enzymes compared with other tissues $(53,54)$. We used these models to assess how oxidative stress responder gene products are affected by chronic in vivo hyperglycemia or insulin resistance.

Only the transcription factor nuclear factor, erythroid-derived 2-like 2 (NFE2L2) was reduced in DT-HG islet grafts in a panel of oxidative stress-related genes (Figure 4A). However, the antioxidant enzymes superoxide dismutase 1 and 2 (SOD1 and SOD2) and glutathione peroxidase 1 (GPX1), as well as NFE2L2, were decreased in HFD+I grafts (Figure 4B). Superoxide levels, as measured by dihydroethidium (DHE) staining, were higher in HFD+I grafts, but they were not changed in DT-HG grafts (Figure 4, C-E).
HFD+I mouse grafts showed no difference in superoxide levels (Figure $4 \mathrm{~F}$ ), indicating that the higher prevailing level of ROS induced by HFD is specific to human islets. These data demonstrate that changes in human islet antioxidant enzyme expression and subsequent increases in ROS are part of the response to chronic insulin resistance and may be a component of the lipotoxic functional consequences of these human grafts. Interestingly, the effect of hyperglycemia and insulin resistance on oxidative stress was different. The insulin resistance of the NSG-HFD model had a greater effect on both antioxidant enzyme expression and ROS levels, suggesting that oxidative stress may be more important as a lipotoxic mechanism than a glucotoxic mechanism.

UPR is not upregulated in response to chronic hyperglycemia or chronic insulin resistance. The efficacy of the UPR influences the ability of islets to meet increased insulin demand under metabolic stressors such as chronic hyperglycemia or insulin resistance 

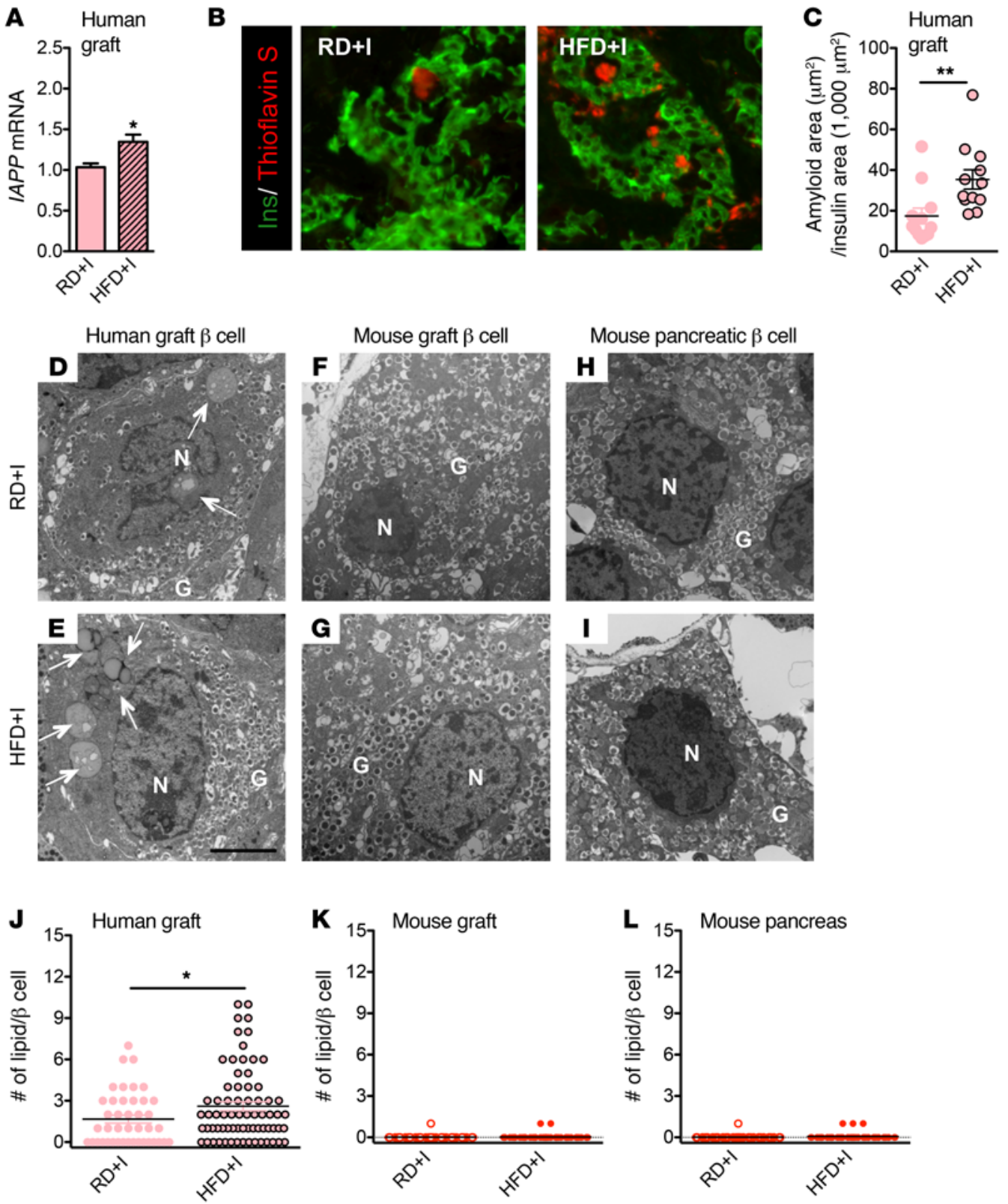

\begin{abstract}
Figure 5. Amyloid deposition in human grafts is increased in NSG-HFD mice. (A) Relative mRNA level of IAPP in human grafts ( $n=5 /$ diet). ${ }^{*} P<0.05$. (B) Representative images of amyloid in human grafts labeled with insulin (green) and thioflavin $\mathrm{S}$ (red). Original magnification, $\times 20$. (C) Measurement of thioflavin $S$ area of human grafts $(n=12$ grafts/diet, $\left.{ }^{* *} P<0.01\right)$. Human, but not mouse, $\beta$ cells accumulate intracellular lipid droplets. Electron microscopy images of $\beta$ cells from human graft (D and $\mathbf{E}$ ), mouse graft ( $\mathbf{F}$ and $\mathbf{G}$ ), and mouse pancreas ( $\mathbf{H}$ and $\mathbf{I}$ ). Arrows point to lipid droplet(s). $\mathrm{N}$, nuclear, $\mathrm{G}$, granule. Scale bar: $3 \mu \mathrm{m}$. (J-L) The number of lipid droplets per $\beta$ cell in human grafts ( $\beta$ cell $n=45-70$ ), mouse grafts $(n=50-71)$, and mouse pancreatic $\beta$ cells $(n=56-74)$. ${ }^{*} P<0.05$. Unpaired 2-tailed Student's $t$ test was used for analysis of statistical significance. Darker bars represent mouse data; lighter bars represent human data.
\end{abstract}

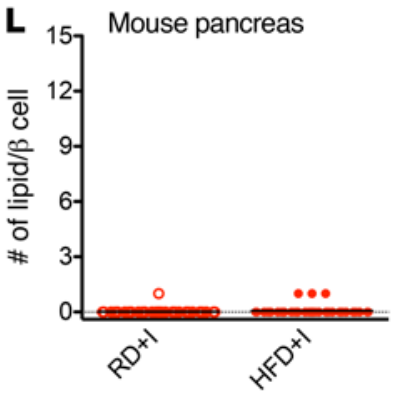

$(55,56)$. To examine the UPR in human islets exposed to chronic hyperglycemia or insulin resistance, we measured the gene expression of 2 chaperones central to the UPR, HSPA5 (GPR78, $\mathrm{BIP}$ ) and HSP9OB1 (GRP94), as well as protein disulfide isomerase family A member 4 (PDIA4) (ERP72). HSPA5 and PDIA4 were increased in DT-NG grafts (Figure $4 G$ ), which successfully maintained normoglycemia, but were unchanged in both DT-HG and HFD+I grafts (Figure 4H), which had impaired insulin secretion. In contrast, pancreatic mouse islets of HFD+I mice, which had robust stimulated insulin secretion, had increased expression of all 3 UPR genes in response to HFD (Figure 4I).

These models demonstrate that islets with preserved stimulated insulin secretion, namely DT-NG human grafts and HFD+I mouse islets, upregulate components of the UPR in contrast with islets with impaired stimulated secretion, namely DT-HG human grafts and HFD+I human grafts. This suggests that inability to stimulate the
UPR may be a glucotoxic and lipotoxic consequence. Alternatively, a lack of UPR induction could be a natural downstream response to either reduced or unchanged insulin transcription and/or translation, in which case this lack of UPR induction would reflect an appropriate homeostatic mechanism, rather than dysfunction. Importantly, mouse and human islets responded similarly.

Chronic insulin resistance, but not chronic hyperglycemia, increases amyloid deposition in human islet grafts. Islet amyloid deposition is a proposed mechanism of human $\beta$ cell dysfunction and death in T2D (57-59). Specifically, it has been proposed that hyperglycemia and HFD promote amyloid formation by increasing cellular stress $(15,16,60)$. To test whether this occurs in human islets exposed to chronic hyperglycemia or insulin resistance in vivo, we measured graft expression of islet amyloid polypeptide (IAPP). Both IAPP expression and amyloid formation were observed in HFD+I grafts (Figure 5A). HFD+I grafts 

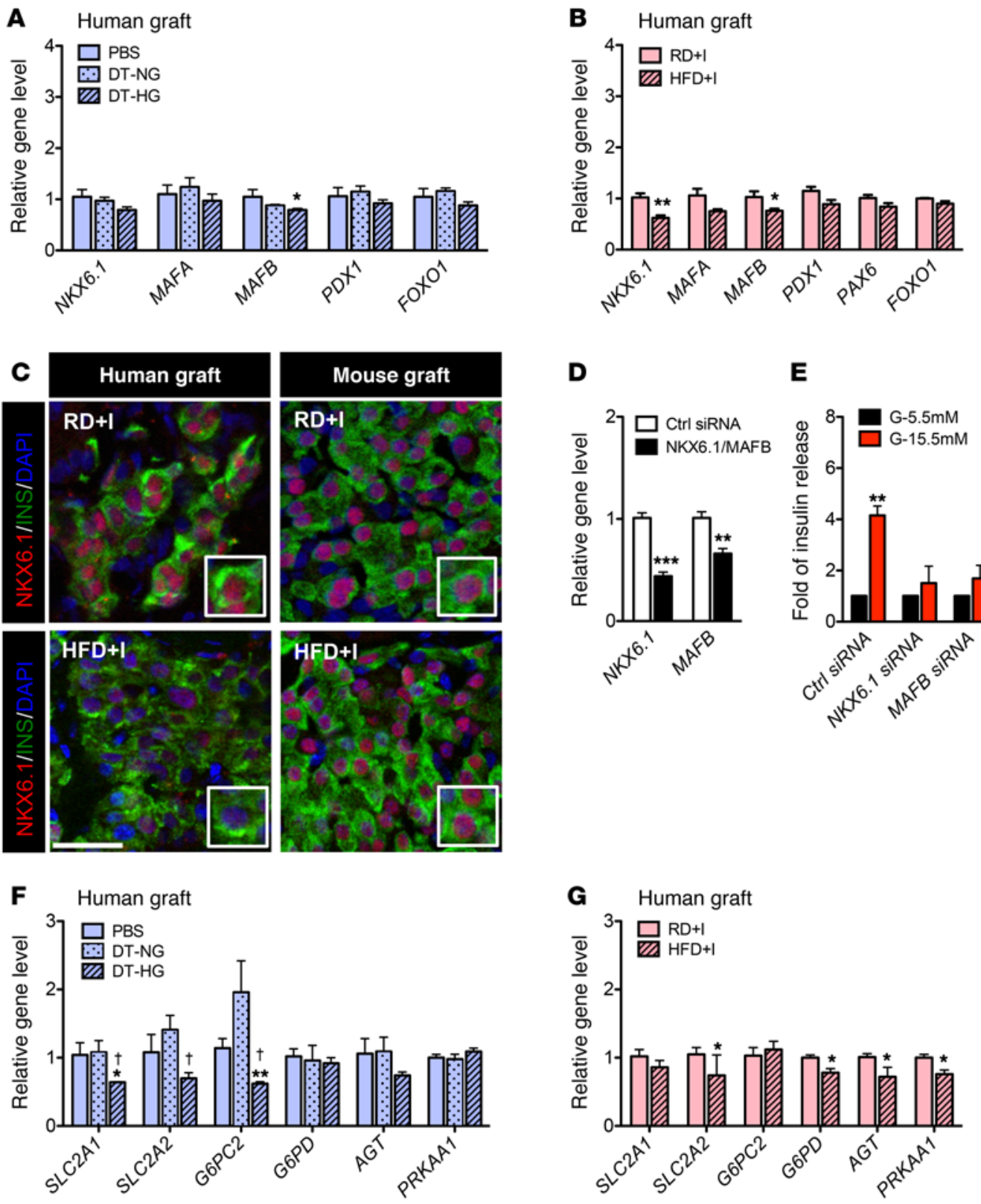
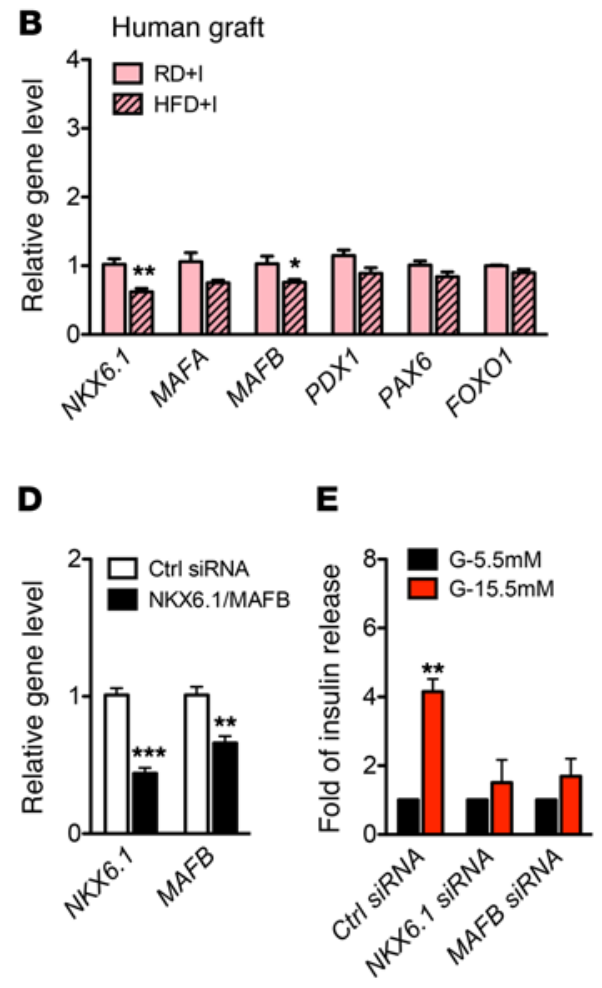

Figure 6. MAFB and NKX6.1 transcription factors and downstream targets are reduced in human islets in DTR and HFD models. (A and B) mRNA levels of transcription factors in human grafts from NSG-DTR (A) or NSG-HFD (B) mice. ${ }^{*} P<0.05,{ }^{* *} P<0.01$, DT-HG vs. PBS or NSG-HFD vs. NSG-RD (PBS, $n=5$, DTR-NG, $n=5$; DTR$\mathrm{HG}, n=10 ; \mathrm{RD}+\mathrm{I}, n=5$; HFD+l, $n=5)$. (C) Representative images of NKX6.1 protein in HFD+I and $\mathrm{RD}+\mathrm{l}$ human grafts (left panels) and mouse grafts (right panels). Scale bar: $50 \mu \mathrm{m}$. (D and E) siRNA knockdown of NKX6.1 or MAFB in EndoC- $\beta \mathrm{H} 1$ cells. (D) Relative level of each gene after treatment with relevant siRNA. ${ }^{* *} P<0.01$, ${ }^{* * *} P<0.001$, relative to control (Ctrl) siRNA for each gene $(n=6$ / gene). (E) Static stimulation of insulin secretion with $5.5 \mathrm{mM}$ or $15.5 \mathrm{mM}$ glucose. ${ }^{* *} P<0.01$, relative to control siRNA, $(n=3$ / group). (F and $\mathbf{G}$ ) mRNA levels of downstream gene targets of MAFB and NKX6.1 in human grafts from NSG-DTR (F) and NSG-HFD (C) mice. ${ }^{*} P<0.05,{ }^{* *} P<0.01$, DT-HG vs. PBS, or NSG-HFD vs. NSG-RD; ${ }^{\dagger} P<0.05$, DT-HG vs. DTNG. Unpaired 2-tailed Student's $t$ test or 1-way ANOVA followed by Newman-Keuls multiple comparison test ( $\mathbf{A}$ and $\mathbf{F}$ ) was used for analysis of statistical significance. Blue represents the NSG-DTR model; red represents the NSGHFD model. Darker bars represent mouse data; lighter bars represent human data. also had larger amyloid deposits than animals fed RD (Figure 5, $\mathrm{B}$ and $\mathrm{C})$. In contrast, there was no change in IAPP expression, amyloid presence, or deposit size in the DT-HG grafts (Supplemental Figure 11). Due to the inherent inability of mouse IAPP to form amyloid (61), mouse grafts were not examined for amyloid. These data suggest that chronic insulin resistance, but not hyperglycemia, is the primary driver of amyloid deposition in human islets. However, this increased islet amyloid deposition did not cause human $\beta$ cell apoptosis (Supplemental Figure 10F).

Human $\beta$ cells exposed to chronic insulin resistance accumulate a greater number of intracellular lipid droplets. Studies have suggested that excess nutrients promote lipid droplet formation within islets (6) and that these lipid droplets affect $\beta$ cell function (62). Using electron microscopy to examine intracellular lipid accumulation, we observed that human $\beta$ cells (Figure 5, D, E and J), but not mouse $\beta$ cells (Figure 5, F-I, K, and L), extensively accumu- lated lipid droplets on RD. Lipid deposition in human $\beta$ cells was also increased in response to HFD (Figure 5J). In addition, normal nontransplanted human pancreatic $\beta$ cells contained lipid droplets (data not shown), suggesting that droplets were not a result of our manipulations. These data indicate that intracellular lipid accumulation is a feature of human, but not mouse, $\beta$ cells and that HFD increases human $\beta$ cell intracellular lipid accumulation.

Chronic insulin resistance and chronic hyperglycemia reduce NKX6.1 and/or MAFB in human $\beta$ cells. Given the increased ROS in HFD+I human islet grafts, we postulated that those $\beta$ cell-enriched transcription factors sensitive to this stressor, specifically MAFA, MAFB, NKX6.1, and/or PDX1, first shown to be affected in mouse models of diabetes and subsequently T2D islets, would be compromised under these circumstances $(28,63)$. MAFB, which is expressed in both human islet $\alpha$ and $\beta$ cells (64), was reduced in both DT-HG human grafts (Figure 6A) and in HFD+I human grafts 


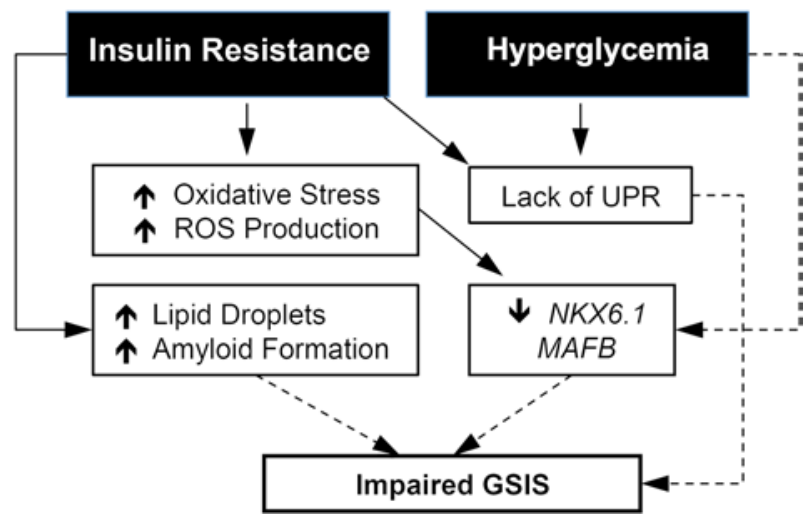

Figure 7. Proposed model of the impaired insulin secretion in transplanted human islets under metabolic stress. Solid lines represent experimental relationships. Dotted lines represent possible relationships.

(Figure 6B) as well as in mouse pancreatic islets in HFD+I mice (Supplemental Figure 12C). Because Mafb is not expressed in adult mouse islet $\beta$ cells (65), this reduction of Mafb in mouse islets is most likely due to a decreased ratio of $\alpha$ to $\beta$ cells, resulting from increased pancreatic $\beta$ cell proliferation (Supplemental Figure 4, F and G). Gene expression and protein levels of NKX6.1, a transcription factor critical to $\beta$ cell identity and function (66-68), were also decreased in HFD+I human grafts (Figure 6C), but were unchanged in mouse grafts and mouse pancreatic islets in the same mice (Figure $6 \mathrm{C}$ and Supplemental Figure 12D). This indicates that human NKX6.1 is more sensitive to HFD-induced insulin resistance than is mouse NKX6.1. Gene expression of MAFA, PDX1, and the panendocrine marker PAX6 was unchanged in DT-HG and HFD+I grafts (Figure 6, A and B, and Supplemental Figure 12, A and B), but mouse pancreatic islets in HFD+I mice had a dramatic increase in Mafa expression (Supplemental Figure 12C). Given the numerous genes regulated by $M a f a$ and $N k x 6.1(66,69,70)$, these gene changes likely mediate the metabolic response in human and mouse islets.

Two MAFA and NKX6.1 targets, INS and GCK, were not changed in HFD+I human islets (Supplemental Figure 13A), but Ins and Gck were increased in mouse islets from the same mice (Supplemental Figure 13C). INS expression was dramatically reduced in DT-HG grafts, compared with both PBS and DT-NG groups, but GCK expression was not (Supplemental Figure 13B). These data suggest that glucotoxic conditions reduce human insulin gene transcription. To ascertain whether decreased NKX6.1 or MAFB affect GSIS in human $\beta$ cells, we performed knockdown experiments in the EndoC- $\beta \mathrm{H} 1$ cell line (71). Reduction of either $M A F B$ or NKX6.1 impaired GSIS (Figure 6, D and E), as observed for MAFB in prior studies $(66,72)$. The expression of various downstream targets of MAFB and NKX6.1 was measured to obtain insight into how changes in their levels could reduce GSIS. We found that SLC2A1 (GLUT1), SLC2A2 (GLUT2), and G6PC2 were significantly reduced in DT-HG human grafts (Figure 6F), whereas SLC2A2 (GLUT2), G6PD, AGT, and PRKAA1 were compromised in HFD+I grafts (Figure 6G). These data strongly suggest that the glucotoxic and lipotoxic changes in human islet function in vivo were at least partially mediated by reduction in the levels of NKX6.1 or MAFB transcription factors (Figure 7).

\section{Discussion}

The terms glucotoxicity, lipotoxicity, and glucolipotoxicity are used frequently to describe a paradigm wherein exposure to excess glucose, lipid, or both directly contribute to islet dysfunction and pathology (1-3). Based on studies in rodent $\beta$ cell lines (4, $12,73)$, human or rodent islets in vitro $(7,52,63)$, and in vivo rodent models $(8,9,28)$, a range of molecular mechanisms, including oxidative stress, ER stress, $\beta$ cell apoptosis, and increased amyloid deposition $(17,18,27,74)$, have been proposed as contributing to these "toxicities." However, there is limited information regarding whether these mechanisms are relevant to human islets in vivo. To address these gaps in our understanding, we generated and/or used mouse models of metabolic stress to study the effect of these conditions on human islets in vivo. These studies demonstrate that chronic and acute hyperglycemia and/or insulin resistance impair stimulated insulin secretion by human islets in vivo. This impairment is similar to observations in human T2D (75) and is not explained by $\beta$ cell death or loss. Chronic insulin resistance decreased human islet antioxidant enzymes, increased superoxide, and decreased the key $\beta$ cell transcription factors NKX6.1 and $M A F B$, while chronic hyperglycemia decreased $M A F B$, but not NKX6.1. Reducing either NKX6.1 or MAFB in a human cell line impaired stimulated insulin secretion, mimicking the functional human islet defects seen in the in vivo models and indicating that reduction of these transcription factors is likely central to the observed defect. Insulin resistance and hyperglycemia potently stimulated mouse $\beta$ cell proliferation, but not human $\beta$ cell proliferation. In addition, HFD increased both islet amyloid and intracellular lipid deposits in human islets. Interestingly, the UPR was not increased in response to either condition, despite increased demand for insulin secretion. Importantly, these studies found that regulatory mechanisms noted in rodent models of T2D or in vitro studies of rodent or human islets were not operative in human islets challenged by chronic hyperglycemia or insulin resistance.

The presence of both human and mouse transplanted islets in the NSG-HFD and NSG-S961 models demonstrated fundamentally different responses of human islets to the same metabolic conditions. These differences included a lack of metabolic stressinduced $\beta$ cell proliferation, decreased insulin gene expression, but unchanged insulin content, accumulation of intracellular lipid droplets, lack of UPR induction, the ROS superoxide in human islets (Figure 4D), and changes in transcription factor expression (Figure 6B). Previous studies have also demonstrated that basal human $\beta$ cell proliferation rates are much lower than those in mouse $(24,76,77)$, that compensatory increases in human $\beta$ cell mass are far smaller than those achieved in mouse (78), and that human $\beta$ cell transcription factor expression profiles are distinct from those in mouse and are not responsive to glucose (35). A recent study proposes that human $\beta$ cell proliferation has been systematically underestimated in postmortem studies due to reduced Ki67 staining in postmortem tissues (79). However, the functional, vascularized state of the transplanted human islets in our studies argues against Ki67-related underestimation of human $\beta$ cell proliferation. In addition, we observed these differences in human and mouse islets across multiple human islet donors. These results highlight the importance of studying human islets and assessing the translational relevance of mouse islet studies. 
As a result of these findings, we propose a paradigm of direct and indirect effects of insulin resistance (excess lipid) and hyperglycemia (excess glucose) on human islets in vivo (Figure 7). In this paradigm, insulin resistance increases the level of ROS, which contributes to reduced expression of the transcription factors NKX6.1 and MAFB. This reduction in transcription factors then impairs stimulated insulin secretion. In this paradigm, hyperglycemia reduces expression of $M A F B$, which impairs stimulated insulin secretion. Other consequences of insulin resistance and/ or hyperglycemia, namely increased islet amyloid formation, increased intracellular lipid droplets, and a lack of UPR stimulation, may contribute to and exacerbate this secretion deficit in the insulin resistance and hyperglycemia models.

In the NSG-HFD model of insulin resistance, the most highly reactive ROS, superoxide, is increased in HFD+I grafts. ROS have been proposed as the mechanism by which excess lipid and hyperglycemia exert many adverse cellular consequences $(52,80)$. ROS are important messengers required for insulin secretion $(81,82)$, but excess nutrients can elevate ROS levels and induce negative secondary consequences. Importantly, superoxide may work in conjunction with other ROS, such as hydrogen peroxide, to reduce NKX6.1 and MAFB expression. In response to excess lipid, the dominant site of lipid oxidation shifts from mitochondria to peroxisomes $(26,27)$. This shift is proposed to result in higher, toxic concentrations of hydrogen peroxide, against which insulin-producing cells have particularly low defenses $(54,83)$. Hydrogen peroxide can then directly reduce expression and/or protein function of NKX6.1, PDX1, and MAFA (28), defining a potential link between the excess lipid of insulin resistance and impaired stimulated insulin secretion. In our NSG-DTR model of chronic hyperglycemia, neither superoxide nor antioxidant expression changed in DTHG grafts. $M A F B$, but not NKX6.1, expression is reduced in this model, suggesting that the type of metabolic stress may influence which transcription factors are affected. The focus of these studies was to examine chronic exposure to moderate, clinically relevant levels of hyperglycemia and insulin resistance. For this reason, analyses of ROS, amyloid, and gene expression were not performed in the $\mathrm{S} 961$ model, which shows acute exposure to extreme metabolic changes. It is possible, however, that these parameters are altered in response to $\$ 961$ treatment.

Our knockdown experiments in EndoC- $\beta \mathrm{H} 1$ cells demonstrate that reduced NKX6.1 or MAFB expression leads to impaired human $\beta$ cell activity (72). Not only is NKX6.1 fundamental to adult $\beta$ cell identity and function (66-68), but knockdown of NKX6.1 in rat INS-1 cells and primary rat islets reduces stimulated insulin secretion without altering basal secretion or insulin content (68). This effect is similar to that seen in the NSG-HFD model, in which NKX6.1 expression is reduced. While human MAFB appears to be important to islet $\beta$ cells (72), mouse MAFB is only essential to mouse islet $\alpha$ cell activity (84). Our results suggest that impaired insulin secretion in HFD+I and DT-HG islets reflects reduced MAFB expression. Interestingly, neither PDX1 nor MAFA, both of which are reduced in human T2D islets (28), is reduced in HFD+I or DT-HG grafts, which may indicate that increased duration and/ or severity of hyperglycemia and/or insulin resistance is required for loss of these particular factors.
In addition to oxidative stress, ER stress has been proposed as a mediator of gluco- and/or lipotoxicity. ER stress can be initiated by chronic activation of the UPR, which is critical for sustaining high levels of insulin production, processing, and packaging (85). Recent work has even suggested that the UPR is a sensor of insulin demand and a regulator of $\beta$ cell mass (86). In our models of insulin resistance or hyperglycemia, the ability to increase stimulated insulin secretion correlates with increased UPR-related gene expression. Lack of UPR induction may functionally compromise the DT-HG and HFD+I human grafts. However, this lack of UPR induction could also be an appropriate response, in which the need for increased human insulin secretion, specifically, is tempered by the shared contribution to secreted insulin by the transplanted HFD + I human $\beta$ cells and the pancreatic mouse $\beta$ cells.

Beyond cellular stress responses, islet amyloid deposition, a pathologic hallmark of human $\mathrm{T} 2 \mathrm{D}$, has been suggested as a mechanism of $\beta$ cell dysfunction and apoptosis $(59,87,88)$. However, studying the development of amyloid in human islets is difficult. The majority of prior data comes either from autopsy studies that do not permit time-course studies or from mouse models that transgenically express human amyloid. Using our models, we found that HFD+I grafts had both more and larger amyloid deposits. Importantly, this increase in amyloid deposition did not lead to increased apoptosis, but could contribute to or reflect the impaired stimulated insulin secretion from human islets. Recent studies suggest that impaired autophagy increases susceptibility to amyloid-related toxicities $(14,89)$, a relationship that can now be examined in human islets using these models.

Our results indicate that $\beta$ cell apoptosis does not contribute to impaired stimulated insulin secretion. Some prior studies demonstrating lipid- and glucose-induced $\beta$ cell death used high concentrations of lipid or glucose in culture (17-20). Importantly, HFD likely generates very different lipid species than the selected lipid moieties of infusion or islet culture studies. Low levels of $\beta$ cell apoptosis and a modest reduction in $\beta$ cell mass are observed in human cadaveric T2D studies $(90,91)$. The duration of metabolic stress experienced by human patients (years or decades) may be required for $\beta$ cell death to occur in vivo, or it may require the coexistence of hyperglycemia and insulin resistance that is present in those patients but not in our chronic models. Alternatively, the combination of very little apoptosis with a decrease in insulin content could point to $\beta$ cell dedifferentiation in response to metabolic stress (92, 93), an intriguing possibility that requires further investigation.

Glucose has been proposed as a mouse $\beta$ cell mitogen $(47,48)$. Both by infusing glucose into human islet graft-containing mice (94) and by using the hyperglycemia of the Akita mouse model (49), modest changes in human $\beta$ cell proliferation rates were noted. However, in our models, hyperglycemia did not stimulate human $\beta$ cell proliferation. Importantly, by cotransplanting mouse islets, we confirmed that mouse $\beta$ cells under the kidney capsule proliferate in response to hyperglycemia or insulin resistance. The lack of increased human $\beta$ cell proliferation in our models is consistent with human autopsy studies of lean, obese, pregnant, and diabetic patients $(95,96)$, although a caveat is that human $\beta$ cells may not respond to in vivo murine stimuli. A previous study of transplanted human islets (97) also showed adaptation of endogenous islets to HFD-induced insulin resistance without 
enhanced human $\beta$ cell proliferation, leaving their observation of increased human graft volume and $\beta$ cell percentage unexplained. The relative age of mouse and human islets must also be considered when interpreting results. Human islets used in our studies were from healthy, nondiabetic, adult donors in the age range in which humans develop T2D. Mouse islets were also from adults, but it is not clear how to control for islet age between these species. Although the NSG genetic background is critical for successful islet engraftment, it also eliminates many islet-immune interactions, which may affect islet function and health (98-100).

This work demonstrates that hyperglycemia and insulin resistance impair stimulated insulin secretion in human islets in vivo and this is at least partly due to reduced expression of NKX6.1 and/or MAFB. In addition, insulin resistance has a broader set of negative consequences than hyperglycemia. Surprisingly, neither hyperglycemia nor insulin resistance stimulated $\beta$ cell proliferation or apoptosis, and the responses of human and mouse islets were fundamentally different in many aspects. Future studies should focus on determining how these abnormalities relate to the clinical phenotypic and genetic subgroups of T2D (101), contribute to the decline in insulin secretion in T2D, and can be therapeutically addressed.

\section{Methods}

Insulin-resistant animal models on an immunodeficient background. Adult male and female (The Jackson Laboratory) B6.Cg-+ $/ L e p^{o b}$ mice were mated with NSG mice (25), and the Lep ${ }^{o b}$ mutation (abbreviated as $o b$ ) was subsequently backcrossed for 10 generations to the NSG strain to create the NSG-ob strain. The colony was maintained by intercrossing NSG $+/ o b$ heterozygotes. These crosses produced NSG-ob/ob mice as well as NSG + $/ o b$ and +/+ WT controls. NSG-ob/ob and NSG-WT controls were studied at 3, 6, and 11 weeks of age. (B6;129 Sv)-Glut4 ${ }^{+/-}$mice were mated with NSG mice and the Glut4 mutation was subsequently backcrossed for 10 generations to the NSG strain to create the NSG-Glut4 strain. The colony was maintained by intercrossing NSG +/Glut4 heterozygotes. These crosses produced NSG-Glut4 ${ }^{-/}$mice as well as NSG-Glut4 ${ }^{+/-}$and Glut $^{+/+}$ WT controls. To create diet-induced, insulin-resistant mice on the immunodeficient background, we fed NSG mice with RD or HFD. Two HFDs were tested: $45 \%$ or $60 \%$ of calories from fat (Research Diets). The $45 \%$ HFD (D12451) contained $45 \%$ of calories from fat, $35 \%$ from carbohydrate, and $20 \%$ from protein. The $60 \%$ HFD (D12492) contained $60 \%$ of calories from fat, $20 \%$ from carbohydrate, and $20 \%$ from protein. The $60 \%$ HFD was used in subsequent studies and was compared with a regular chow diet (Lab Diet, cata$\log 5001$ ), which contained $13.5 \%$ of calories from fat, $58 \%$ from carbohydrate, and $28.5 \%$ from protein.

Hyperglycemic animal model on an immunodeficient background. NSG-Tg(Ins2-HBEGF)6832)Ugfm/Sz mice, referred to as NSG RIPDTR mice, were developed by backcrossing the RIP-DTR transgene from a B6;CBA-RIP-DTR stock provided by Pedro Herrera. The original B6;CBA Tg(Ins2-HBEGF)6832)Ugfm/Sz mice were made by injecting the construct into B6;CBA eggs. The transgene was backcrossed using a marker-assisted speed congenic method to the NOD.Cg-Prkdcsid $I l 2 \mathrm{rg}^{\text {tmlWjl}} / \mathrm{SzJ}$ (abbreviated as NOD-scid IL2r $\gamma^{\text {null }}$ or NSG) strain background. These NSG RIP-DTR mice express the human DTR driven by a RIP. The RIP-DTR transgene was then fixed to homozygosity and maintained as a homozygous line. Transgenic mice, with 2 autosomal copies of the RIP-DTR transgene (40), in which DTR expression is driven by the RIP, were backcrossed onto the NSG background (25) for more than 10 generations, resulting in the NSG-DTR mouse.

DT preparation and administration. DT (List Biological Laboratories Inc., catalog 150) was administered to NSG-DTR mice in a single, $300 \mu \mathrm{l}$ i.p. injection of $0.5,1.0,2.5,5.0,10$, or 25 ng total DT. Control NSG-DTR mice (PBS) were treated with an equal volume of $1 \times$ PBS (Sigma-Aldrich). All animals in a cohort (with human islets from the same human donor) were injected with DT or PBS on the same day. Stock solutions reconstituted with water were stored at $-20^{\circ} \mathrm{C}$, according to the manufacturer's recommendations, and stock solution aliquots were diluted for each use.

S961 treatment. S961 reagent $(44,45)$ was provided by Lauge Schäffer (Novo Nordisk). S961 is a 43-aa peptide antagonist that induces many consequences of insulin resistance in rodents, including hyperglycemia, hyperinsulinemia, decreased hepatic glycogen storage, and decreased adipocyte triglyceride storage $(44,45)$. The affinity of S961 for the insulin receptor rivals that of insulin itself, and specificity of $\mathrm{S} 961$ for the insulin receptor, rather than the similar IGF receptor, exceeds that of insulin (44). Either S961 or 1× PBS was loaded into Alzet 2001 (20 nM) osmotic pumps. Pumps were implanted 2 weeks after human islet engraftment. Animals were sacrificed, and tissues were harvested at either 7 or 14 days after pump implantation.

Human and mouse islets. Human islets ( $n=13$ preparations, Supplemental Table 1) were obtained from islet isolation centers that are part of the Integrated Islet Distribution Network (http://iidp.coh.org/). Assessment of human islet function was performed by perifusion on the day of islet arrival, as previously described $(35,102)$. Sixty size-matched islets were perifused with $5.6 \mathrm{mM}$ glucose, $16.7 \mathrm{mM}$ glucose, and 16.7 mM glucose with $100 \mu \mathrm{M} 3$-isobutyl-1-methyl-xanthine (IBMX) (SigmaAldrich, catalog I5879-1G). Insulin secretion was normalized to IEQs (102). Mouse islets were isolated from 13- to 15-week-old NSG mice or C57BL/6J mice (Jackson Laboratory) as described (41).

Islet transplantation. NSG or NSG-DTR male mice, between 12 and 20 weeks of age, were used for transplantation (103). For the NSG-HFD model, each recipient mouse received 1,500 IEQ human islets, 140 islets isolated from NSG mice, or 200 islets from C57BL/6J mice transplanted under the kidney capsule. After 2 weeks engraftment, the mice were placed on an RD or a 60\% HFD for 12 weeks. For the NSG-DTR model, each recipient mouse received 2,000 or 4,000 human islet IEQ. For the $\$ 961$ model, each recipient mouse received 4,000 human islet IEQ or 200 islets from C57BL/6J mice. All data with human islets from all models were normalized to 2,000 transplanted IEQ.

Immunohistochemistry of pancreas and human and mouse grafts. Immunohistochemical studies were performed as described $(33,104$, 105). Antibodies used in this study were as follows: guinea pig antihuman insulin (catalog 0564, DAKO), rabbit anti-human Ki67 (catalog ab15580, Abcam), rabbit anti-human PDX1 (from Christopher Wright, Vanderbilt University), rabbit anti-human NKX6.1 ( $\beta$ Cell Biology Consortium), mouse anti-human CD31, and rat anti-mouse PECAM (cata$\log 550389$, catalog 550274, BD Biosciences - Pharmingen). Images were acquired with an Olympus BX41 fluorescence microscope or a confocal laser-scanning microscope (PDX1 and NKX6.1 stain).

Assessment of glucose tolerance, random blood glucose, serum insulin, triglyceride, cholesterol, and tissue insulin content. The i.p. glucose tolerance tests were performed after a 6-hour fast, as previously described (104). All measurements were taken at the same recurring time of day. 
Serum human insulin was measured using a species-specific radioimmunoassay from Millipore (catalog RI-14K). Serum mouse insulin was calculated as the difference between total (mouse and rodent, crossreactive with human) and human-specific insulin measurements. Total serum insulin, pancreatic insulin content, and graft insulin content were measured as previously described (105). Serum triglyceride and cholesterol levels were measured from $10 \mu \mathrm{l}$ of plasma using commercially available kits (Raichem, Cliniqa) as previously described (106).

Assessment of apoptosis, oxidative stress, and amyloid deposition. Apoptosis was assessed by TUNEL stain (catalog S7165, Millipore) according to the manufacturer's instructions. DHE (catalog D7008, SigmaAldrich) was used to measure $\mathrm{O}_{2}^{-}$in cryosections, as described $(107,108)$. The cryosections were washed 3 times by PBS, followed by DHE staining for 30 minutes. Fluorescence intensity of islet grafts was quantified using Image J software (107). To assess amyloid deposits, sections from human grafts were first stained with the insulin antibody and then incubated with $0.5 \%$ concentration Thioflavin S (catalog T-1892, Sigma-Aldrich) in PBS. Grafts were visualized, and the images were obtained using an Olympus BX41 fluorescence microscope. Thioflavin S and insulin-positive areas were then quantified with MetaMorph software.

$q R T-P C R$. Total RNA from human grafts and mouse islets was isolated using an RNAqueous RNA Isolation Kit (catalog 1912, Ambion), as previously described (35). RNA bioanalysis and quality control and quantity assessment (QC/QA), assessed in the Vanderbilt Function Genomics Shared Resource (FGSR) core lab, showed a 28S/18S ratio greater than 1.2 and a RNA integrity number (RIN) greater than 8.2. Quantitative reverse-transcriptase PCR (qRT-PCR) was performed using the TaqMan primer-probe and reagents from Applied Biosystems as described (35). $A C T B, T B P$, and TFRC were used as endogenous control genes. Relative changes in mRNA expression were calculated by the comparative $\Delta \mathrm{Ct}$ method using the Applied Biosystems StepOnePlus System. Primers are listed in Supplemental Table 2.

Electron microscopy. Ultrastructure of $\beta$ cells and vasculature were studied by transmission electron microscopy (41). Mouse pancreas and grafts were removed after perfusion and fixed in $2.5 \%$ gluteraldehyde in $0.1 \mathrm{M}$ cacodylate buffer. Samples were subsequently imaged on the Philips/FEI Tecnai T12 microscope at various magnifications.

siRNA-mediated knockdown of NKX6.1 and MAFB in EndoC- $\beta H 1$ cells. Knockdown of NKX6.1 and MAFB was accomplished 3 days prior to GSIS using the Dharmafect no. 1 reagent following the manufacturer's protocol. Briefly, ON-TARGETplus Smartpool siRNA against human NKX6.1 (catalog L-020083-00), human MAFB (catalog L-00901800; GE Dharmacon), and scrambled nontargeting siRNA (catalog D001810; GE Dharmacon) were introduced into $2 \times 10^{6}$ EndoC- $\beta \mathrm{H} 1$ cells (71) in antibiotic-free media. Following an overnight incubation, the cells were grown in normal growth media for an additional 36 hours and then overnight in low-glucose medium (1.1 mM glucose, 2\% BSA, $50 \mu \mathrm{M}$ 2-mercaptoethanol, $10 \mathrm{mM}$ nicotinamide, $5.5 \mu \mathrm{g} / \mathrm{ml}$ transferrin,
$6.7 \mathrm{ng} / \mathrm{ml}$ selenite, and penicillin-streptomycin at 100 units $/ \mathrm{ml})$. Cells were incubated for 1 hour in DMEM base medium supplemented with $5.5 \mathrm{mM}$ glucose or $15.5 \mathrm{mM}$ glucose. Secreted insulin was analyzed from culture medium and was normalized to the insulin content following cell lysis (cell lysis buffer: $1 \mathrm{M}$ Tris, Triton X-100, glycerol, $5 \mathrm{M} \mathrm{NaCl}, 0.2$ M EGTA, protease inhibitor tablet). Insulin levels were analyzed by the Vanderbilt Hormone Assay Core. To assess silencing effects of NKX6.1 and MAFB, qRT-PCR was performed using TaqMan primers.

Statistics. Results are shown as mean \pm SEM. Two-tailed Student's $t$ test or 1-way ANOVA was used for analysis of statistical significance. ANOVAs were followed by Newman-Keuls multiple comparison tests. A $P$ value of less than 0.05 was considered significant.

Study approval. Studies with human islets were approved by the Vanderbilt Institutional Review Board. All animal studies were approved by the Vanderbilt Institutional Animal Care and Use Committee.

\section{Author contributions}

NSK, CD, RS, and ACP designed experiments and wrote the manuscript. CD, NSK, GP, AS, CT, RA, MS, and IGP performed experiments related to islet transplantation studies in NSG-DTR, NSG-HFD, and NSG-S961 models and characterization of the $\mathrm{NSG}-\mathrm{Glut}^{-/-}$and NSG-ob/ob models. CE and JMS performed lipid quantification experiments. HAC and RS performed human cell line experiments. LDS, DLG, and PLH backcrossed the B6;CBARIP-DTR stock onto the NSG background, generating NSG-DTR mice, and edited the manuscript.

\section{Acknowledgments}

We thank Janice Williams for her expertise and effort in acquiring electron microscopy images. This work was supported by grants from the Department of Veterans Affairs (BX000666, BX002223), the National Institute of Diabetes and Digestive and Kidney Diseases (DK68854, DK66636, DK69603, DK63439, DK62641, DK72473, DK89572, DK89538, DK68764, DK92758, DK97829, DK94199, DK104211, DK050203, DK090570, DK89572, DK104218, DK106755, DK104211, DK108120), JDRF, the Vanderbilt Diabetes Research and Training Center (DK20593), and the Helmsley Charitable Trust (2012PG-T1D018 and 2015PGT1D057). The EndoC- $\beta$ H1 cell line was provided by Raphael Scharfmann and Philippe Ravassard and the S961 was provided by Lauge Schäffer. N.S. Kayton was supported by a Vanderbilt Molecular Endocrinology Training Program grant (5T32 DK07563).

Address correspondence to: Alvin C. Powers, Division of Diabetes, Endocrinology, and Metabolism, Vanderbilt University School of Medicine, 7465 Medical Research Building IV, 2215 Garland Avenue, Nashville, Tennessee 37232-0475, USA. Phone: 615.936.7678; E-mail al.powers@vanderbilt.edu.
1. Unger RH, Zhou YT. Lipotoxicity of beta-cells in obesity and in other causes of fatty acid spillover. Diabetes. 2001;50(suppl 1):S118-S121.

2. Poitout V, Robertson RP. Glucolipotoxicity: fuel excess and beta-cell dysfunction. Endocr Rev. 2008;29(3):351-366.

3. Poitout V, et al. Glucolipotoxicity of the pancreatic $\beta$ cell. Biochim Biophys Acta. 2010;1801(3):289-298.

4. Gleason CE, Gonzalez M, Harmon JS, Robert- son RP. Determinants of glucose toxicity and its reversibility in the pancreatic islet $\beta$-cell line, HIT-T15. Am J Physiol Endocrinol Metab. 2000;279(5):E997-E1002.

5. Dubois M, et al. Glucotoxicity inhibits late steps of insulin exocytosis. Endocrinology. 2007;148(4):1605-1614.

6. Vernier S, et al. $\beta$-Cell metabolic alterations under chronic nutrient overload in rat and human islets.
Islets. 2012;4(6):379-392.

7. Olofsson CS, et al. Long-term exposure to glucose and lipids inhibits glucose-induced insulin secretion downstream of granule fusion with plasma membrane. Diabetes. 2007;56(7):1888-1897.

8. Goh TT, et al. Lipid-induced $\beta$-cell dysfunction in vivo in models of progressive $\beta$-cell failure. Am J Physiol Endocrinol Metab. 2007;292(2):E549-E560.

9. Tang C, et al. Susceptibility to fatty acid-induced 
$\beta$-cell dysfunction is enhanced in prediabetic diabetes-prone biobreeding rats: a potential link between $\beta$-cell lipotoxicity and islet inflammation. Endocrinology. 2013;154(1):89-101.

10. Kelpe CL, et al. Palmitate inhibition of insulin gene expression is mediated at the transcriptional level via ceramide synthesis. J Biol Chem. 2003;278(32):30015-30021.

11. Poitout $\mathrm{V}$, et al. Regulation of the insulin gene by glucose and fatty acids. J Nutr. 2006;136(4):873-876.

12. Moran A, et al. Differentiation of glucose toxicity from beta cell exhaustion during the evolution of defective insulin gene expression in the pancreatic islet cell line, HIT-T15. J Clin Invest. 1997;99(3):534-539.

13. Cao P, et al. Islet amyloid: from fundamental biophysics to mechanisms of cytotoxicity. FEBS Lett. 2013;587(8):1106-1118.

14. Shigihara N, et al. Human IAPP-induced pancreatic $\beta$ cell toxicity and its regulation by autophagy. JClin Invest. 2014;124(8):3634-3644.

15. Hull RL, et al. Increased dietary fat promotes islet amyloid formation and $\beta$-cell secretory dysfunction in a transgenic mouse model of islet amyloid. Diabetes. 2003;52(2):372-379.

16. Verchere CB, et al. Islet amyloid formation associated with hyperglycemia in transgenic mice with pancreatic $\beta$ cell expression of human islet amyloid polypeptide. Proc Natl Acad Sci U S A. 1996;93(8):3492-3496.

17. Shimabukuro M, et al. Lipoapoptosis in $\beta$-cells of obese prediabetic fa/fa rats. Role of serine palmitoyltransferase overexpression. J Biol Chem. 1998;273(49):32487-32490.

18. Federici M, et al. High glucose causes apoptosis in cultured human pancreatic islets of Langerhans: a potential role for regulation of specific Bcl family genes toward an apoptotic cell death program. Diabetes. 2001;50(6):1290-1301.

19. Lupi R, et al. Prolonged exposure to free fatty acids has cytostatic and pro-apoptotic effects on human pancreatic islets: evidence that $\beta$-cell death is caspase mediated, partially dependent on ceramide pathway, and Bcl-2 regulated. Diabetes. 2002;51(5):1437-1442.

20. Véret J, et al. Ceramide synthase 4 and de novo production of ceramides with specific $\mathrm{N}$-acyl chain lengths are involved in glucolipotoxicityinduced apoptosis of INS-1 $\beta$-cells. Biochem J. 2011;438(1):177-189.

21. Boslem E, Meikle PJ, Biden TJ. Roles of ceramide and sphingolipids in pancreatic $\beta$-cell function and dysfunction. Islets. 2012;4(3):177-187.

22. Prentki M, Joly E, El-Assaad W, Roduit R. Malonyl-CoA signaling, lipid partitioning, and glucolipotoxicity: role in $\beta$-cell adaptation and failure in the etiology of diabetes. Diabetes. 2002;51(suppl 3):S405-S413.

23. Fiaschi-Taesch NM, et al. Cytoplasmic-nuclear trafficking of G1/S cell cycle molecules and adult human $\beta$-cell replication: a revised model of human $\beta$-cell G1/S control. Diabetes. 2013;62(7):2460-2470.

24. Stewart AF, et al. Human $\beta$-cell proliferation and intracellular signaling: part 3. Diabetes. 2015;64(6):1872-1885.

25. Shultz LD, Ishikawa F, Greiner DL. Humanized mice in translational biomedical research. Nat
Rev Immunol. 2007;7(2):118-130.

26. Gehrmann W, Elsner M, Lenzen S. Role of metabolically generated reactive oxygen species for lipotoxicity in pancreatic $\beta$-cells. Diabetes Obes Metab. 2010;12(suppl 2):149-158.

27. Elsner M, Gehrmann W, Lenzen S. Peroxisomegenerated hydrogen peroxide as important mediator of lipotoxicity in insulin-producing cells. Diabetes. 2011;60(1):200-208.

28. Guo $S$, et al. Inactivation of specific $\beta$ cell transcription factors in type 2 diabetes. JClin Invest. 2013;123(8):3305-3316.

29. Matsuoka TA, et al. Preserving Mafa expression in diabetic islet $\beta$-cells improves glycemic control in vivo. J Biol Chem. 2015;290(12):7647-7657.

30. Nair G, Hebrok M. ScienceDirectIslet formation in mice and men: lessons for the generation of functional insulin-producing. Curr Opin Genet Dev. 2015;32:171-180.

31. Brissova M, et al. Intraislet endothelial cells contribute to revascularization of transplanted pancreatic islets. Diabetes. 2004;53(5):1318-1325.

32. Negi S, Jetha A, Aikin R, Hasilo C, Sladek R, Paraskevas S. Analysis of $\beta$-cell gene expression reveals inflammatory signaling and evidence of dedifferentiation following human islet isolation and culture. PLoS One. 2012;7(1):e30415.

33. Brissova $\mathrm{M}$, et al. Assessment of human pancreatic islet architecture and composition by laser scanning confocal microscopy. J Histochem Cytochem. 2005;53(9):1087-1097.

34. Cabrera O, et al. The unique cytoarchitecture of human pancreatic islets has implications for islet cell function. Proc Natl Acad Sci U S A. 2009;315(4):683-696.

35. Dai C, et al. Islet-enriched gene expression and glucose-induced insulin secretion in human and mouse islets. Diabetologia. 2012;55(3):707-718.

36. Greiner DL, et al. Humanized mice for the study of type 1 and type 2 diabetes. Ann N Y Acad Sci. 2011;1245:55-58.

37. Brehm MA, et al. Human immune system development and rejection of human islet allografts in spontaneously diabetic NOD-Rag1null IL2rgammanull Ins2Akita mice. Diabetes. 2010;59(9):2265-2270.

38. Brehm MA, Powers AC, Shultz LD, Greiner DL. Advancing animal models of human type 1 diabetes by engraftment of functional human tissues in immunodeficient mice. Cold Spring Harb Perspect Med. 2012;2(5):a007757.

39. Brehm MA, Wiles MV, Greiner DL, Shultz LD. Generation of improved humanized mouse models for human infectious diseases. J Immunol Methods. 2014;410:3-17.

40. Thorel F, et al. Conversion of adult pancreatic [agr]-cells to [bgr]-cells after extreme [bgr]-cell loss. Nature. 2010;464(7292):1149-1154.

41. Dai C, et al. Pancreatic islet vasculature adapts to insulin resistance through dilation and not angiogenesis. Diabetes. 2013;62(12):4144-4153.

42. Mosser RE, et al. High-fat diet-induced $\beta$-cell proliferation occurs prior to insulin resistance in C57Bl/6J male mice. Am J Physiol Endocrinol Metab. 2015;308(7):E573-E582.

43. Mezza T, Kulkarni RN. The regulation of pre- and post-maturational plasticity of mammalian islet cell mass. Diabetologia. 2014;57(7):1291-1303.
44. Schäffer L, et al. A novel high-affinity peptide antagonist to the insulin receptor. Biochem Biophys Res Commun. 2008;376(2):380-383.

45. Vikram A, Jena G. S961, an insulin receptor antagonist causes hyperinsulinemia, insulin-resistance and depletion of energy stores in rats. Biochem Biophys Res Commun. 2010;398(2):260-265.

46. Mezza T, et al. Insulin resistance alters islet morphology in nondiabetic humans. Diabetes. 2014;63(3):994-1007.

47. Porat $\mathrm{S}$, et al. Control of pancreatic $\beta$ cell regeneration by glucose metabolism. Cell Metab. 2011;13(4):440-449.

48. Alonso LC, et al. Glucose infusion in mice: a new model to induce beta-cell replication. Diabetes. 2007;56(7):1792-1801.

49. Diiorio P, et al. Hyperglycemia-induced proliferation of adult human beta cells engrafted into spontaneously diabetic immunodeficient NODRag1null IL2rynull Ins2Akita mice. Pancreas. 2011;40(7):1147-1149.

50. Yi P, Park JS, Melton DA. Betatrophin: a hormone that controls pancreatic $\beta$ cell proliferation. Cell. 2013;153(4):747-758.

51. Jiao Y, Le Lay J, Yu M, Naji A, Kaestner KH. Elevated mouse hepatic betatrophin expression does not increase human $\beta$-cell replication in the transplant setting. Diabetes. 2014;63(4):1283-1288.

52. Robertson RP. Chronic oxidative stress as a central mechanism for glucose toxicity in pancreatic islet $\beta$ cells in diabetes. J Biol Chem. 2004;279(41):42351-42354.

53. Welsh $\mathrm{N}$, et al. Differences in the expression of heat-shock proteins and antioxidant enzymes between human and rodent pancreatic islets: implications for the pathogenesis of insulin-dependent diabetes mellitus. Mol Med. 1995;1(7):806-820.

54. Tiedge M, Lortz S, Drinkgern J, Lenzen S. Relation between antioxidant enzyme gene expression and antioxidative defense status of insulinproducing cells. Diabetes. 1997;46(11):1733-1742.

55. Chan JY, Luzuriaga J, Bensellam M, Biden TJ, Laybutt DR. Failure of the adaptive unfolded protein response in islets of obese mice is linked with abnormalities in $\beta$-cell gene expression and progression to diabetes. Diabetes. 2013;62(5):1557-1568.

56. Cadavez L, et al. Chaperones ameliorate beta cell dysfunction associated with human islet amyloid polypeptide overexpression. PLoS One. 2014;9(7):e101797.

57. Kahn SE, Andrikopoulos S, Verchere CB. Islet amyloid: a long-recognized but underappreciated pathological feature of type 2 diabetes. Diabetes. 1999;48(2):241-253.

58. Citri A, et al. Amyloid oligomers in diabetic and nondiabetic human pancreas. Transl Res. 2009;153(1):24-32.

59. Potter KJ, et al. Islet amyloid deposition limits the viability of human islet grafts but not porcine islet grafts. Proc Natl Acad Sci US A. 2010;107(9):4305-4310.

60. Qi D, et al. Fatty acids induce amylin expression and secretion by pancreatic $\beta$-cells. Am J Physiol Endocrinol Metab. 2010;298(1):E99-E107.

61. Tokuyama T, et al. Expression of human islet amyloid polypeptide/amylin impairs insulin secretion in mouse pancreatic $\beta$ cells. Metab Clin 
Exp. 1997;46(9):1044-1051.

62. Trevino MB, et al. Perilipin 5 regulates islet lipid metabolism and insulin secretion in a cAMPdependent manner: implication of its role in the postprandial insulin secretion. Diabetes. 2015;64(4):1299-1310.

63. Harmon JS, Stein R, Robertson RP. Oxidative stress-mediated, post-translational loss of MafA protein as a contributing mechanism to loss of insulin gene expression in glucotoxic $\beta$ cells. J Biol Chem. 2005;280(12):11107-11113.

64. Dorrell C, et al. Transcriptomes of the major human pancreatic cell types. Diabetologia. 2011;54(11):2832-2844.

65. Artner I, et al. MafB is required for islet $\beta$ cell maturation. Proc Natl Acad Sci U S A. 2007;104(10):3853-3858.

66. Taylor BL, Liu FF, Sander M. Nkx6.1 is essential for maintaining the functional state of pancreatic $\beta$ cells. Cell Rep. 2013;4(6):1262-1275.

67. Schaffer AE, et al. Nkx6.1 controls a gene regulatory network required for establishing and maintaining pancreatic $\beta$ cell identity. PLoS Genet. 2013;9(1):e1003274.

68. Schisler JC, et al. The Nkx6.1 homeodomain transcription factor suppresses glucagon expression and regulates glucose-stimulated insulin secretion in islet $\beta$ cells. Proc Natl Acad Sci U S A. 2005;102(20):7297-7302.

69. Matsuoka T-A et al. Members of the large Maf transcription family regulate insulin gene transcription in islet $\beta$ cells. Mol Cell Biol. 2003;23(17):6049-6062.

70. Artner I, et al. MafA and MafB regulate genes critical to beta-cells in a unique temporal manner. Diabetes. 2010;59(10):2530-2539.

71. Ravassard $\mathrm{P}$, et al. A genetically engineered human pancreatic $\beta$ cell line exhibiting glucose-inducible insulin secretion. J Clin Invest. 2011;121(9):3589-3597.

72. Scoville DW, et al. MLL3 and MLL4 methyltransferases bind to the MAFA and MAFB transcription factors to regulate islet $\beta$-cell function. Diabetes. 2015;64(11):3772-3783.

73. Pino MF, et al. Elevated glucose attenuates human insulin gene promoter activity in INS-1 pancreatic $\beta$-cells via reduced nuclear factor binding to the A5/core and Z element. Mol Endocrinol. 2005;19(5):1343-1360.

74. Laybutt DR, et al. Endoplasmic reticulum stress contributes to beta cell apoptosis in type 2 diabetes. Diabetologia. 2007;50(4):752-763.

75. Elder DA, Prigeon RL, Wadwa RP, Dolan LM, D'Alessio DA. Beta-cell function, insulin sensitivity, and glucose tolerance in obese diabetic and nondiabetic adolescents and young adults. J Clin Endocrinol Metab. 2006;91(1):185-191.

76. Tyrberg B, Ustinov J, Otonkoski T, Andersson A. Stimulated endocrine cell proliferation and differentiation in transplanted human pancreatic islets: effects of the ob gene and compensatory growth of the implantation organ. Diabetes. 2001;50(2):301-307.

77. Kulkarni RN, Mizrachi E-B, Ocana AG, Stewart
AF. Human $\beta$-cell proliferation and intracellular signaling: driving in the dark without a road map. Diabetes. 2012;61(9):2205-2213.

78. Rahier J, Guiot Y, Goebbels RM, Sempoux C, Henquin JC. Pancreatic beta-cell mass in European subjects with type 2 diabetes. Diabetes Obes Metab. 2008;10(suppl 4):32-42.

79. Sullivan BA, Hollister-Lock J, Bonner-Weir S, Weir GC. Reduced Ki67 staining in the postmortem state calls into question past conclusions about the lack of turnover of adult human $\beta$-cells. Diabetes. 2015;64(5):1698-1702.

80. Evans JL, Goldfine ID, Maddux BA, Grodsky GM. Oxidative stress and stress-activated signaling pathways: a unifying hypothesis of type 2 diabetes. Endocr Rev. 2002;23(5):599-622.

81. Pi J, et al. Reactive oxygen species as a signal in glucose-stimulated insulin secretion. Diabetes. 2007;56(7):1783-1791.

82. Leloup C, et al. Mitochondrial reactive oxygen species are obligatory signals for glucose-induced insulin secretion. Diabetes. 2009;58(3):673-681.

83. Lenzen S, Drinkgern J, Tiedge M. Low antioxidant enzyme gene expression in pancreatic islets compared with various other mouse tissues. Free Radic Biol Med. 1996;20(3):463-466.

84. Conrad E, et al. The MAFB transcription factor impacts islet $\alpha$-cell function in rodents and represents a unique signature of primate islet $\beta$-cells. $A m$ JPhysiol Endocrinol Metab. 2016;310(1):E91-E102.

85. Papa FR. Endoplasmic reticulum stress, pancreatic $\beta$-cell degeneration, and diabetes. Cold Spring Harb Perspect Med. 2012;2(9):a007666.

86. Sharma RB, et al. Insulin demand regulates $\beta$ cell number via the unfolded protein response. J Clin Invest. 2015;125(10):3831-3846.

87. Butler AE, Janson J, Soeller WC, Butler PC. Increased beta-cell apoptosis prevents adaptive increase in $\beta$-cell mass in mouse model of type 2 diabetes: evidence for role of islet amyloid formation rather than direct action of amyloid. Diabetes. 2003;52(9):2304-2314.

88. Poojari C, Xiao D, Batista VS, Strodel B. Membrane permeation induced by aggregates of human islet amyloid polypeptides. Biophys J. 2013;105(10):2323-2332.

89. Rivera JF, Costes S, Gurlo T, Glabe CG, Butler PC. Autophagy defends pancreatic $\beta$ cells from human islet amyloid polypeptide-induced toxicity. J Clin Invest. 2014;124(8):3489-3500.

90. Butler AE, et al. $\beta$-Cell deficit and increased betacell apoptosis in humans with type 2 diabetes. Diabetes. 2003;52(1):102-110.

91. Butler AE, et al. Diabetes due to a progressive defect in $\beta$-cell mass in rats transgenic for human islet amyloid polypeptide (HIP Rat): a new model for type 2 diabetes. Diabetes. 2004;53(6):1509-1516

92. Talchai $C$, et al. Pancreatic $\beta$ cell dedifferentiation as a mechanism of diabetic $\beta$ cell failure. Cell. 2012;150(6):1223-1234.

93. Wang Z, York NW, Nichols CG, Remedi MS. Pancreatic $\beta$ cell dedifferentiation in diabetes and redifferentiation following insulin therapy. Cell Metab. 2014;19(5):872-882.

94. Levitt HE, et al. Glucose stimulates human $\beta$ cell replication in vivo in islets transplanted into NOD-severe combined immunodeficiency (SCID) mice. Diabetologia. 2011;54(3):572-582.

95. Saisho $Y$, et al. $\beta$-Cell mass and turnover in humans: effects of obesity and aging. Diabetes Care. 2013;36(1):111-117.

96. Butler AE, et al. Adaptive changes in pancreatic $\beta$ cell fractional area and beta cell turnover in human pregnancy. Diabetologia. 2010;53(10):2167-2176

97. Gargani S, et al. Adaptive changes of human islets to an obesogenic environment in the mouse. Diabetologia. 2013;56(2):350-358.

98. Imai Y, et al. Interaction between cytokines and inflammatory cells in islet dysfunction, insulin resistance and vascular disease. Diabetes Obes Metab. 2013;15(suppl 3):117-129.

99. Donath MY, Dalmas E, Sauter NS, Böni-Schnetzler M. Inflammation in obesity and diabetes: islet dysfunction and therapeutic opportunity. Cell Metab. 2013;17(6):860-872.

100.Wu Y, et al. Chronic inflammation exacerbates glucose metabolism disorders in C57BL/6J mice fed with high-fat diet. J Endocrinol. 2013;219(3):195-204.

101.Li L, et al. Identification of type 2 diabetes subgroups through topological analysis of patient similarity. Sci Transl Med. 2015;7(311):311ra174.

102. Kayton NS, et al. Human islet preparations distributed for research exhibit a variety of insulinsecretory profiles. Am J Physiol Endocrinol Metab 2015;308(7):E592-E602.

103. Brissova $\mathrm{M}$, et al. Islet microenvironment, modulated by vascular endothelial growth factor-A signaling, promotes $\beta$ cell regeneration. Cell Metab. 2014;19(3):498-511.

104. Reinert RB, et al. Vascular endothelial growth factor-a and islet vascularization are necessary in developing, but not adult, pancreatic islets. Diabetes. 2013;62(12):4154-4164.

105. Cai Q, et al. Enhanced expression of VEGF-A in $\beta$ cells increases endothelial cell number but impairs islet morphogenesis and $\beta$ cell proliferation. Dev Biol. 2012;367(1):40-54.

106. Cappel DA, et al. Cholesteryl ester transfer protein protects against insulin resistance in obese female mice. Mol Metab. 2013;2(4):457-467.

107. Kang L, et al. Heterozygous SOD2 deletion impairs glucose-stimulated insulin secretion, but not insulin action, in high-fat-fed mice. Diabetes. 2014;63(11):3699-3710.

108. Cheng Q, Law PK, de Gasparo M, Leung PS. Combination of the dipeptidyl peptidase IV inhibitor LAF237 [(S)-1-[(3-hydroxy-1-adamantyl)ammo] acetyl-2-cyanopyrrolidine] with the angiotensin II type 1 receptor antagonist valsar$\tan$ [N-(1-oxopentyl)-N-[[2'-(1H-tetrazol-5-yl)[1,1'-biphenyl]-4-yl]methyl]-L-valine] enhances pancreatic islet morphology and function in a mouse model of type 2 diabetes. J Pharmacol Exp Ther. 2008;327(3):683-691. 